九州大学学術情報リポジトリ

Kyushu University Institutional Repository

Hermitian symmetric spaces of tube type and multivariate Meixner-Pollaczek polynomials

Faraut, Jacques

Institut de Mathématiques de Jussieu, Université Pierre et Marie Curie

Wakayama, Masato

Faculty of Mathematics, Kyushu University

http://hdl. handle. net/2324/12558

出版情報 : MI Preprint Series. 2008-13，2008-12-06. 九州大学大学院数理学研究院 バージョン：

権利関係 : 


\section{Preprint Series}

Kyushu University

The Grobal COE Program

Math-for-Industry Education \& Research Hub

Hermitian symmetric spaces of tube type and multivariate Meixner-Pollaczek polynomials

\section{J. Faraut \& M. Wakayama}

MI 2008-13

( Received December 6, 2008 )

Faculty of Mathematics

Kyushu University

Fukuoka, JAPAN 


\title{
HERMITIAN SYMMETRIC SPACES OF TUBE TYPE
}

\section{AND MULTIVARIATE MEIXNER-POLLACZEK POLYNOMIALS}

\author{
Jacques Faraut \& Masato Wakayama
}

ABSTRACT. Harmonic analysis on Hermitian symmetric spaces of tube type is a natural framework for introducing multivariate Meixner-Pollaczek polynomials. Their main properties are established in this setting: generating and determinantal formulae, difference equations. As an application we consider the problem of evaluating moments related to a multivariate Barnes type integral involving the Harish-Chandra $c$-function of a symmetric cone.

In this paper we revisit the identity

$$
\left(\frac{1+x}{1-x}\right)^{n}=1+2 \sum_{m=0}^{\infty} c(m, n) x^{m+1},
$$

where

$$
c(m, n)=\sum_{k=0}^{m}\left(\begin{array}{c}
m \\
k
\end{array}\right)\left(\begin{array}{c}
n \\
k+1
\end{array}\right),
$$

for $n \in \mathbb{Z}$. The coefficients $c(m, n)$ appear in the formula giving the moments of the statistical distribution $\mu_{n}$ of the eigenvalues of a random Hermitian matrix in case of the Gauss unitary ensemble (GUE):

$$
\mathfrak{M}_{2 m}\left(\mu_{n}\right)=\frac{1}{n} \int_{H e r m(n, \mathbb{C})} \operatorname{tr}\left(X^{2 m}\right) \mathbb{P}_{n}(d X),
$$

where $\mathbb{P}_{n}$ is a Gaussian probability on the space $\operatorname{Herm}(n, \mathbb{C})$ of $n \times n$ Hermitan matrices:

$$
\mathbb{P}_{n}(d X)=C_{n} e^{-\operatorname{tr}\left(X^{2}\right)} \lambda(d X)
$$

( $\lambda$ is the Lebesgue measure). In fact

$$
\mathfrak{M}_{2 m}\left(\mu_{n}\right)=\frac{1}{n} \frac{(2 m) !}{2^{m} m !} c(m, n) .
$$

See [Harer-Zagier,1986], §4, [Mehta,1991], formula 5.5.30, [Haagerup-Thorbjørnsen,2003], $\S 4$, [Faraut,2004], §5. We will see how this identity appears in the framework of harmonic 
analysis on the unit disc and upper halfplane, and that the coefficients $c(m, n)$ are essentially Meixner-Pollaczek polynomials. In [Davidson-Ólafsson-Zhang,2003] a generalization of this formula appears in the framework of Hermitian symmetric spaces of tube type. We will consider such an extension by using a different method.

Let us describe the scheme of the paper in the one variable case. We start from weighted Bergman spaces on the unit disc. The Cayley transform maps these spaces to weighted Bergman spaces on the right half plane. Then, by an inverse Laplace transform, we obtain an $L^{2}$-space on the positive half-line. Finally we perform a Mellin transform and get an $L^{2}$-space on $\mathbb{R}$. Simultaneously, starting from the monomials on the disc, we obtain on each step an orthogonal basis, and, at the last step, the Meixner-Pollaczek polynomials as, essentially, Mellin transforms of Laguerre polynomials. In this way, starting from the binomial formula, seen as a generating formula for the monomials, one obtains a generating formula for the Meixner-Pollaczek polynomials. Similarly, from the fact that the monomials are eigenfunctions of the Euler operator, it follows that the Meixner-Pollaczek polynomials are eigenfunctions of a difference operator. This scheme extends almost word for word in the framework of Hermitian symmetric spaces of tube type, and leads to a natural definition of the multivariate Meixner-Pollaczek polynomials and their properties. In this extension the positive half-line becomes a symmetric cone, and the Mellin transform generalizes as a spherical Fourier transform.

In the first section we consider the one variable case, to make clear the scheme which is carried on in the general case. In Section 2 we develop our construction in the setting of Hermitian symmetric spaces of tube type. The invariant differential operators on a symmetric cone turn out to be a powerful tool. Multivariate Laguerre polynomials, which appear in the third step of the construction, have been considered with the same point of view in [Davidson-Ólafsson,2003], [Aristidou-Davidson-Ólafsson,2006] and [2007] (see also [Chébli-Faraut,2004]). Eventually multivariate Meixner-Pollaczek show up as, essentially, spherical Fourier transforms of multivariate Laguerre polynomials. Similar analysis has been considered in [Ørsted-Zhang,1994], related to invariant differential operators on a Hermitian symmetric space. This construction leads to a generating formula, as it is shown in Section 3. In case of the multiplicity $d=2$, we establish in Section 4 determinantal formulae for multivariate Laguerre and Meixner-Pollaczek polynomials. We show in Section 5 that multivariate Meixner-Pollaczek polynomials are solutions of a difference equation, and satisfy a Pieri's formula. In the last section an application is given to the evaluation of multivariate moments related to a Barnes type integral involving the Harish-Chandra $c$-function of a symmetric cone, yielding an alternative proof of a result by Mimachi [1999a].

It is quite natural to develop this analysis in the framework of the special functions associated to root systems. This point of view is considered in [Sahi-Zhang,2007].

Part of this work has been done as the first author was visiting the Faculty of Mathematics of Kyushu University. This author would like to thank the colleagues of this faculty for their hospitality.

Mathematics Subject Index: 32M15, 33C45, 43A90

Keywords: Meixner-Pollaczek polynomial, Laguerre polynomial, Hermitian symmetric space, Jordan algebra, spherical function. 
1. Functions spaces on the unit disc and the complex half-plane, one variable Meixner-Pollaczek polynomials. - In the first part of the paper we will consider the one dimensional case, and develop some classical analysis on weighted Bergman spaces on the unit disc $\mathcal{D}$ in $\mathbb{C}$, and on the right halfplane $T$, and then about Laplace integral representation of holomorphic functions and Mellin transform. Finally Meixner-Pollaczek polynomials will show up in this setting.

(1) For $\nu>1$ let $\mathcal{H}_{\nu}^{2}(\mathcal{D})$ denote the Hilbert space of holomorphic functions $f$ on the unit disc $\mathcal{D}=\{w \in \mathbb{C}|| w \mid<1\}$ such that

$$
\|f\|_{\nu}^{2}=a_{\nu}^{(1)} \int_{\mathcal{D}}|f(w)|^{2}\left(1-|w|^{2}\right)^{\nu-2} m(d w)<\infty
$$

where $m$ is the Lebesgue measure on $\mathbb{C}$. The constant $a_{\nu}=\frac{\nu-1}{\pi}$ is choosen such that the function $\phi_{0} \equiv 1$ has norm 1 . The monomials $\phi_{m}(w)=w^{m}$ form an orthogonal basis of $\mathcal{H}_{\nu}^{2}(\mathcal{D})$, and

$$
\left\|\phi_{m}\right\|_{\nu}^{2}=\frac{m !}{(\nu)_{m}}
$$

(2) The Cayley transform

$$
w \mapsto z=c(w)=\frac{1+w}{1-w}
$$

maps the unit disc $\mathcal{D}$ onto the right half-plane $T=\{z=x+i y \in \mathbb{C} \mid x>0\}$, and its inverse is given by

$$
c^{-1}(z)=\frac{z-1}{z+1} .
$$

For a holomorphic function $f$ on $\mathcal{D}$, define the function $F=C_{\nu} f$ on $T$ by

$$
F(z)=\left(C_{\nu} f\right)(z)=\left(\frac{z+1}{2}\right)^{-\nu} f\left(\frac{z-1}{z+1}\right) .
$$

The map $C_{\nu}$ is a unitary isomorphism from $\mathcal{H}_{\nu}^{2}(\mathcal{D})$ onto the space $\mathcal{H}_{\nu}^{2}(T)$ of holomorphic functions $F$ on $T$ such that

$$
\|F\|_{\nu}^{2}=a_{\nu}^{(2)} \int_{T}|F(x+i y)|^{2} x^{\nu-2} d x d y<\infty .
$$

The constant $a_{\nu}^{(2)}$ is such that the function $F_{0}^{(\nu)}, F_{0}^{(\nu)}(z)=\left(\frac{z+1}{2}\right)^{-\nu}$, image of $\phi_{0}$, has norm 1. The functions

$$
F_{m}^{(\nu)}=C_{\nu} \phi_{m}, \quad F_{m}^{(\nu)}(z)=\left(\frac{z+1}{2}\right)^{-\nu}\left(\frac{z-1}{z+1}\right)^{m}
$$


form an orthogonal basis of $\mathcal{H}_{\nu}^{2}(T)$, and

$$
\left\|F_{m}^{(\nu)}\right\|_{\nu}^{2}=\frac{m !}{(\nu)_{m}}
$$

(3) The functions in $\mathcal{H}_{\nu}^{2}(T)$ admit Laplace integral representations. Define the modified Laplace transform $\mathcal{L}_{\nu}$ of a function $\psi$ on $] 0, \infty[$ by

$$
\left(\mathcal{L}_{\nu} \psi\right)(z)=a_{\nu}^{(3)} \int_{0}^{\infty} e^{-z u} \psi(u) u^{\nu-1} d u
$$

with $a_{\nu}^{(3)}=\frac{2^{\nu}}{\Gamma(\nu)}$. For $\psi \in L_{\nu}^{2}(0, \infty)$, equipped with the norm given by

$$
\|\psi\|_{\nu}^{2}=a_{\nu}^{(3)} \int_{0}^{\infty}|\psi(u)|^{2} u^{\nu-1} d u,
$$

the function $\mathcal{L}_{\nu} \psi$ is holomorphic on $T$, and $\mathcal{L}_{\nu}$ is a unitary isomorphism from $L_{\nu}^{2}(0, \infty)$ onto $\mathcal{H}_{\nu}^{2}(T)$. In particular, if $\psi_{0}(u)=e^{-u}$, then $\mathcal{L}_{\nu} \psi_{0}=F_{0}$, and $\psi_{0}$ has norm 1. If $\psi(u)=e^{-u} u^{k}$, then

$$
\left(\mathcal{L}_{\nu} \psi\right)(z)=\frac{2^{\nu}}{\Gamma(\nu)} \int_{0}^{\infty} e^{-(z+1) u} u^{\nu+k-1} d u=2^{-k}(\nu)_{k}\left(\frac{2}{z+1}\right)^{\nu+k} .
$$

Define the Laguerre functions as

$$
\psi_{m}^{(\nu)}(u)=e^{-u} L_{m}^{(\nu-1)}(2 u),
$$

where $L_{m}^{(\alpha)}$ denotes the classical Laguerre polynomial of degree $m$ :

$$
L_{m}^{(\nu-1)}(x)=\sum_{k=1}^{m} \frac{\Gamma(m+\nu)}{\Gamma(k+\nu)} \frac{(-x)^{k}}{k !(m-k) !}=\frac{(\nu)_{m}}{m !} \sum_{k=0}^{m}\left(\begin{array}{c}
m \\
k
\end{array}\right) \frac{1}{(\nu)_{k}}(-x)^{k} .
$$

Hence

$$
\psi_{m}^{(\nu)}(u)=\frac{(\nu)_{m}}{m !} \sum_{k=0}^{m}(-2)^{k}\left(\begin{array}{c}
m \\
k
\end{array}\right) \frac{1}{(\nu)_{k}} e^{-u} u^{k} .
$$

Let us compute the modified Laplace transform of the Laguerre functions:

$$
\left(\mathcal{L}_{\nu} \psi_{m}^{(\nu)}\right)(z)=\frac{(\nu)_{m}}{m !} \sum_{k=0}^{m}(-1)^{k}\left(\begin{array}{c}
m \\
k
\end{array}\right)\left(\frac{2}{z+1}\right)^{\nu+k}=\frac{(\nu)_{m}}{m !} F_{m}^{(\nu)}(z)
$$

by the binomial formula. It follows that the Laguerre functions $\psi_{m}^{(\nu)}$ form an orthogonal basis of $L_{\nu}^{2}(0, \infty)$, and that

$$
\left\|\psi_{m}^{(\nu)}\right\|_{\nu}^{2}=\frac{(\nu)_{m}}{m !} .
$$


(4) Finally we perform a Mellin transform. We define the modified Mellin transform of a function $\psi$ on $] 0, \infty[$ as

$$
\mathcal{M}_{\nu} \psi(s)=\frac{1}{\Gamma\left(s+\frac{\nu}{2}\right)} \int_{0}^{\infty} \psi(u) u^{s+\frac{\nu}{2}-1} d u .
$$

By the classical Plancherel theorem

$$
\int_{0}^{\infty}|\psi(u)|^{2} u^{\nu-1} d u=\frac{1}{2 \pi} \int_{-\infty}^{\infty}\left|\mathcal{M}_{\nu} \psi(i \lambda)\right|^{2}\left|\Gamma\left(i \lambda+\frac{\nu}{2}\right)\right|^{2} d \lambda,
$$

and $\mathcal{M}_{\nu}$ is a unitary isomorphism from $L_{\nu}^{2}(0, \infty)$ onto $L^{2}\left(\mathbb{R}, M(d \lambda)\right.$, with $M(d \lambda)=a_{\nu}^{(4)}\left|\Gamma\left(i \lambda+\frac{\nu}{2}\right)\right|^{2} d \lambda$. The constant $a_{\nu}^{(4)}$ is such that the function $\mathcal{M}_{\nu} \psi_{0} \equiv 1$ has norm 1 :

$$
a_{\nu}^{(4)}=\frac{1}{2 \pi} \frac{2^{\nu}}{\Gamma(\nu)}
$$

If $\psi(u)=e^{-u} u^{k}$, then $\mathcal{M}_{\nu} \psi(s)=\left(s+\frac{\nu}{2}\right)_{k}$ is a polynomial of degree $k$. We define the polynomials $q_{m}^{(\nu)}$ as the modified Mellin transform of the Laguerre functions $\psi_{m}^{(\nu)}$ : $q_{m}^{(\nu)}=\mathcal{M}_{\nu} \psi_{m}^{(\nu)}$. The polynomials $q_{m}^{(\nu)}$, when restricted to the line $i \mathbb{R}$, are orthogonal with respect to the measure $M(d \lambda)$. Hence their zeros are located on the imaginary axis. Furthermore

$$
\left\|q_{m}^{(\nu)}\right\|_{\nu}^{2}:=\int_{-\infty}^{\infty}\left|q_{m}^{\nu}(i \lambda)\right|^{2} M(d \lambda)=\frac{(\nu)_{m}}{m !}
$$

The polynomials $q_{m}^{(\nu)}$ are essentially Meixner-Pollaczek polynomials. Recall the hypergeometric representation of the Meixner-Pollaczek polynomials (see [Andrews-AskeyRoy,1999], p.348):

$$
P_{m}^{\alpha}(\lambda ; \phi)=\frac{(2 \alpha)_{m}}{m !} e_{2}^{i m \phi} F_{1}\left(-m, \alpha+i \lambda ; 2 \alpha ; 1-e^{-2 i \phi}\right) .
$$

Proposition 1.1.

$$
q_{m}^{(\nu)}(s)=\frac{(\nu)_{m}}{m !}{ }_{2} F_{1}\left(-m, s+\frac{\nu}{2} ; \nu ; 2\right), \quad \text { or } \quad q_{m}^{(\nu)}(i \lambda)=(-i)^{m} P_{m}^{\frac{\nu}{2}}\left(\lambda ; \frac{\pi}{2}\right) .
$$

Furthermore one can write

$$
q_{m}^{(\nu)}(s)=\sum_{k=0}^{m} 2^{k}\left(\begin{array}{c}
m+\nu-1 \\
m-k
\end{array}\right)\left(\begin{array}{c}
-s-\frac{\nu}{2} \\
k
\end{array}\right)
$$

Proof. Recall that

$$
\psi_{m}^{(\nu)}(u)=\frac{(\nu)_{m}}{m !} \sum_{k=0}^{m}(-2)^{k}\left(\begin{array}{c}
m \\
k
\end{array}\right) \frac{1}{(\nu)_{k}} e^{-u} u^{k}
$$


Therefore

$$
q_{m}^{(\nu)}(s)=\frac{(\nu)_{m}}{m !} \sum_{k=0}^{m}(-2)^{k}\left(\begin{array}{c}
m \\
k
\end{array}\right) \frac{1}{(\nu)_{k}}\left(s+\frac{\nu}{2}\right)_{k}=\frac{(\nu)_{m}}{m !}{ }_{2} F_{1}\left(-m, s+\frac{\nu}{2} ; \nu ; 2\right) .
$$

Observe further that

$$
\left(s+\frac{\nu}{2}\right)_{k}=(-1)^{k} k !\left(\begin{array}{c}
-s-\frac{\nu}{2} \\
k
\end{array}\right) .
$$
$q_{m}^{(\nu)}$.

This construction provides a generating formula for the Meixner-Pollaczek polynomials

Proposition 1.2. - For $\nu, s \in \mathbb{C}, w \in \mathbb{C}$ with $|w|<1$,

$$
\sum_{m=0}^{\infty} q_{m}^{(\nu)}(s) w^{m}=(1-w)^{s-\frac{\nu}{2}}(1+w)^{-s-\frac{\nu}{2}}
$$

Proof. We may assume $\operatorname{Re} \nu>0$, and $\operatorname{Re}\left(s+\frac{\nu}{2}\right)>0$. The statement for $\nu, s \in \mathbb{C}$ will then follows by analytic continuation. Define, for $|w|<1,|\zeta|<1$,

$$
H_{\nu}^{(1)}(w, \zeta)=\sum_{m=0}^{\infty} \frac{(\nu)_{m}}{m !} \zeta^{m} w^{m}
$$

By the classical binomial expansion,

$$
H_{\nu}^{(1)}(w, \zeta)=(1-w \zeta)^{-\nu}
$$

We apply now the transform $C_{\nu}$ to both handsides with respect to $\zeta$ and get

$$
H_{\nu}^{(2)}(w, z)=\sum_{m=0}^{\infty} \frac{(\nu)_{m}}{m !} F_{m}^{(\nu)}(z) w^{m}
$$

and

$$
H_{\nu}^{(2)}(w, z)=2^{\nu}(1-w)^{-\nu}(z+c(w))^{-\nu} .
$$

Next we apply the inverse of the modified Laplace transform $\mathcal{L}_{\nu}$ with respect ot $z$ :

$$
H_{\nu}^{(3)}(w, u)=\sum_{m=0}^{\infty} \psi_{m}^{(\nu)}(u) w^{m},
$$

and

$$
H_{\nu}^{(3)}(w, u)=(1-w)^{-\nu} e^{-u \frac{1+w}{1-w}} .
$$


Finally we apply the modified Mellin transform $\mathcal{M}_{\nu}$ with respect ot $u$ :

$$
H_{\nu}^{(4)}(w, s)=\sum_{m=0}^{\infty} q_{m}^{(\nu)}(s) w^{m}
$$

and

$$
H_{\nu}^{(4)}(w, s)=(1-w)^{s-\frac{\nu}{2}}(1+w)^{-s-\frac{\nu}{2}}
$$

Since $H_{\nu}^{(4)}(-w, s)=H_{\nu}^{(4)}(w,-s)$, it follows that $q_{m}^{(\nu)}$ has the parity of $m: q_{m}^{(\nu)}(-s)=$ $(-1)^{m} q_{m}^{(\nu)}(s)$.

For $\nu=0$ this gives

$$
\left(\frac{1-w}{1+w}\right)^{s}=\sum_{m=0}^{\infty}{ }_{2} F_{1}(-m, s ; 0 ; 2) w^{m}
$$

and, with $s=-n$, one gets the identity in the introduction:

$$
\left(\frac{1+w}{1-w}\right)^{n}=1+2 \sum_{m=0}^{\infty} c(m, n) w^{m+1}
$$

In this setting we obtain also a difference equation for the Meixner-Pollaczek polynomials $q_{m}^{(\nu)}$, and a three terms relation. Let $D_{\nu}$ be the following difference operator:

$$
D_{\nu} f(s)=\left(s+\frac{\nu}{2}\right) f(s+1)-\left(s-\frac{\nu}{2}\right) f(s-1) .
$$

Proposition 1.3. - The Meixner-Pollaczek polynomials $q_{m}^{(\nu)}$ are eigenfunctions of the operator $D_{\nu}$ :

$$
D_{\nu} q_{m}^{(\nu)}=(2 m+\nu) q_{m}^{(\nu)},
$$

and satisfie the following three terms relation: for $m \geq 1$,

$$
2 s q_{m}^{(\nu)}(s)=(m+\nu-1) q_{m-1}^{(\nu)}(s)-(m+1) q_{m+1}^{(\nu)}(s) .
$$

Proof. a) Let us consider on the disc $\mathcal{D}$ the modified Euler operator $D_{\nu}^{(1)}=2 w \frac{d}{d w}+\nu$. The monomials $\phi_{m}(w)=w^{m}$ are eigenfunctions of it: $D_{\nu}^{(1)} \phi_{m}=(2 m+\nu) \phi_{m}$.

Then on the half-plane $T$ consider the operator $D_{\nu}^{(2)}$ such that $C_{\nu} D_{\nu}^{(1)}=D_{\nu}^{(2)} C_{\nu}$. Then

$$
D_{\nu}^{(2)}=\left(z^{2}-1\right) \frac{d}{d z}+\nu z, \quad D_{\nu}^{(2)} F_{m}^{(\nu)}=(2 m+\nu) F_{m}^{(\nu)},
$$

since $F_{m}^{(\nu)}=C_{\nu} \phi_{m}$ 
Further consider the operator $D_{\nu}^{(3)}$ on $] 0, \infty\left[\right.$ such that $D_{\nu}^{(2)} \mathcal{L}_{\nu}=\mathcal{L}_{\nu} D_{\nu}^{(3)}$. Then

$$
D_{\nu}^{(3)}=-u \frac{d^{2}}{d u^{2}}-\nu \frac{d}{d u}+u, \quad D_{\nu}^{(3)} \psi_{m}^{(\nu)}=(2 m+\nu) \psi_{m}^{(\nu)}
$$

since $\mathcal{L}_{\nu} \psi_{m}^{(\nu)}=F_{m}^{(\nu)}$.

Finally let the operator $D_{\nu}^{(4)}$ on $\mathbb{C}$ be such that $\mathcal{M}_{\nu} D_{\nu}^{(3)}=D_{\nu}^{(4)} \mathcal{M}_{\nu}$. Then $D_{\nu}^{(4)}=D_{\nu}$, and, since $\mathcal{M}_{\nu} \psi_{m}^{(\nu)}=q_{m}^{(\nu)}$, we get

$$
D_{\nu} q_{m}^{(\nu)}=(2 m+\nu) q_{m}^{(\nu)} .
$$

b) Proposition 1.2 can be written, with $s=m+\frac{\nu}{2}$,

$$
\sum_{k=0}^{\infty} q_{k}^{(\nu)}\left(m+\frac{\nu}{2}\right) w^{k}=(1-w)^{m}(1+w)^{-m-\nu}=(-1)^{m} 2^{-\nu} F_{m}^{(\nu)}(w) .
$$

Let us apply the operator $D_{\nu}^{(2)}$ to both sides. Since $D_{\nu}^{(2)}\left(w^{k}\right)=(k+\nu) w^{k+1}-k w^{k-1}$, and $D_{\nu}^{(2)} F_{m}^{(\nu)}=(2 m+\nu) F_{m}^{(\nu)}$, we get, for $k \geq 1$ :

$$
(2 m+\nu) q_{k}^{(\nu)}\left(m+\frac{\nu}{2}\right)=(k+\nu-1) q_{k-1}^{(\nu)}\left(m+\frac{\nu}{2}\right)-(k+1) q_{k+1}^{(\nu)}\left(m+\frac{\nu}{2}\right)
$$

Notice that the operators $D_{\nu}^{(i)}(i=1,2,3,4)$ are essentially selfadjoint in corresponding Hilbert spaces (with appropriate domains).

The fact that the zeros of the polynomials $q_{m}^{(\nu)}$ are located on the imaginary axis is related to the so-called Local Riemann hypothesis (see [Bump-Choi-Kurlberg-Vaaler,2000]). In a recent paper by Kuznetsov $([2008])$ the three term relation is used to study the expansion of the Riemann $\Xi$-function in series of Meixner-Pollaczek polynomials, and the zeros of the partial sums are investigated.

2. Function spaces on a Hermitian symmetric space of tube type and multivariate Meixner-Pollaczek polynomials. - Let $\mathcal{D}$ be an irreducible Hermitian symmetric domain of tube type realized as the unit ball in a simple complex Jordan algebra $V_{\mathbb{C}}$ of dimension $N$ and rank $n$, seen as the complexification of a Euclidean Jordan algebra $V$. The Cayley transform

$$
w \mapsto z=c(w)=(e+w)(e-w)^{-1}
$$

maps the domain $\mathcal{D}$ onto the tube domain $T_{\Omega}=\left\{z=x+i y \in V_{\mathbb{C}} \mid x \in \Omega\right\}$, where $\Omega$ denotes the symmetric cone in $V$. Let $G$ be the identity component in the group $G(\Omega)$ of linear automorphisms of $\Omega$, and $K \subset G$ the isotropy subgroup of the unit element $e \in V$.

The space $\mathcal{P}\left(V_{\mathbb{C}}\right)$ of holomorphic polynomials on $V_{\mathbb{C}}$ decomposes multiplicity free as

$$
\mathcal{P}\left(V_{\mathbb{C}}\right)=\bigoplus_{\mathbf{m}} \mathcal{P}_{\mathbf{m}}
$$


where $\mathcal{P}_{\mathbf{m}}$ is a finite dimensional subspace, irreducible under $G$. The parameter $\mathbf{m}$ is a partition $\mathbf{m}=\left(m_{1}, \ldots, m_{n}\right) \in \mathbb{N}^{n}, m_{1} \geq \cdots \geq m_{n}$. The subspace $\mathcal{P}_{\mathbf{m}}^{K}$ of $K$-invariant polynomials in $\mathcal{P}_{\mathbf{m}}$ is one dimensional, generated by the spherical polynomial $\Phi_{\mathbf{m}}$, normalized by the condition $\Phi_{\mathbf{m}}(e)=1$. We consider on $\mathcal{P}\left(V_{\mathbb{C}}\right)$ the Fischer inner product, and denote by $K^{\mathbf{m}}\left(w_{1}, w_{2}\right)$ the reproducing kernel of $\mathcal{P}_{\mathbf{m}}$. (See [Faraut-Korányi,1994], Chapter XI.)

The Gindikin gamma function $\Gamma_{\Omega}$ of the symmetric cone $\Omega$ is defined, for $\mathbf{s} \in \mathbb{C}^{n}$, with $\operatorname{Re} s_{j}>\frac{d}{2}(j-1)$, by

$$
\Gamma_{\Omega}(\mathbf{s})=\int_{\Omega} e^{-\operatorname{tr}(x)} \Delta_{\mathbf{s}}(x) \Delta(x)^{-\frac{N}{n}} m(d x),
$$

where $\Delta$ is the determinant on $V_{\mathbb{C}}, \Delta_{\mathbf{S}}$ is the power function defined on $\Omega$, and the multiplicity $d$ is such that $N=n+\frac{d}{2} n(n-1)$. (Notation $m$ will denote the Euclidean measure on any Euclidean vector space.) Its evaluation gives

$$
\Gamma_{\Omega}(\mathbf{s})=(2 \pi)^{\frac{N-n}{2}} \prod_{j=1}^{n} \Gamma\left(s_{j}-\frac{d}{2}(j-1)\right),
$$

hence $\Gamma_{\Omega}$ extends analytically as a meromorphic function on $\mathbb{C}^{n}$.

(See [Faraut-Korányi,1994], Chapter VII.) For $\alpha \in \mathbb{C}$, and a partition $\mathbf{m}$, one writes $\alpha+\mathbf{m}=\left(\alpha+m_{1}, \ldots, \alpha+m_{n}\right) \in \mathbb{C}$, and defines the generalized Pochhammer symbol

$$
(\alpha)_{\mathbf{m}}=\frac{\Gamma_{\Omega}(\alpha+\mathbf{m})}{\Gamma_{\Omega}(\alpha)} .
$$

For $\nu>2 \frac{N}{n}-1, \mathcal{H}_{\nu}^{2}(\mathcal{D})$ denotes the Hilbert space of holomorphic functions $f$ on $\mathcal{D}$ such that

$$
\|f\|_{\nu}^{2}=a_{\nu}^{(1)} \int_{\mathcal{D}}|f(w)|^{2} h(w)^{\nu-2 \frac{N}{n}} m(d w)<\infty .
$$

Recall that $h\left(w_{1}, w_{2}\right)$ denotes a polynomial on $V_{\mathbb{C}} \times V_{\mathbb{C}}$, holomorphic in $w_{1}$, antiholomorphic in $w_{2}$, such that, for $u \in V, h(u, u)=\Delta\left(e-u^{2}\right)$, and, for $w \in V_{\mathbb{C}}, h(w)=h(w, w)$ by definition. The constant $a_{\nu}^{(1)}$ is such that the function $\Phi_{0} \equiv 1$ has norm 1 . The spherical polynomials $\Phi_{\mathbf{m}}$ form an orthogonal basis of the space $\mathcal{H}_{\nu}^{2}(\mathcal{D})^{K}$ of $K$-invariant functions in $\mathcal{H}_{\nu}^{2}(\mathcal{D})$, and

$$
\left\|\Phi_{\mathbf{m}}\right\|_{\nu}^{2}=\frac{1}{d_{\mathbf{m}}} \frac{\left(\frac{N}{n}\right)_{\mathbf{m}}}{(\nu)_{\mathbf{m}}}
$$

where $d_{\mathbf{m}}=\operatorname{dim} \mathcal{P}_{\mathbf{m}}$.

For a function $f$ holomorphic on $\mathcal{D}$, one defines the function $F=C_{\nu} f$ on $T_{\Omega}$ by

$$
F(z)=\left(C_{\nu} f\right)(z)=\Delta\left(\frac{z+e}{2}\right)^{-\nu} f\left((z-e)(z+e)^{-1}\right) .
$$

The map $C_{\nu}$ is a unitary isomorphism from $\mathcal{H}_{\nu}^{2}(\mathcal{D})$ onto the space $\mathcal{H}_{\nu}^{2}\left(T_{\Omega}\right)$ of holomorphic functions $F$ on $T_{\Omega}$ such that

$$
\|F\|_{\nu}^{2}=a_{\nu}^{(2)} \int_{T_{\Omega}}|F(z)|^{2} \Delta(x)^{\nu-2 \frac{N}{n}} m(d z)<\infty .
$$


The constant $a_{\nu}^{(2)}$ is such that the function $F_{0}^{(\nu)}=C_{\nu} \Phi_{0}, F_{0}^{(\nu)}(z)=\Delta\left(\frac{z+e}{2}\right)^{-\nu}$, has norm 1. The functions $F_{\mathbf{m}}^{(\nu)}=C_{\nu} \Phi_{\mathbf{m}}$ form an orthogonal basis of $\mathcal{H}_{\nu}^{2}\left(T_{\Omega}\right)^{K}$ of $K$-invariant functions in $\mathcal{H}_{\nu}^{2}\left(T_{\Omega}\right)$.

The functions in $\mathcal{H}_{\nu}^{2}\left(T_{\Omega}\right)$ admit a Laplace integral representation. The modified Laplace transform $\mathcal{L}_{\nu}$ given, for a function $\psi$ on $\Omega$, by

$$
\left(\mathcal{L}_{\nu} \psi\right)(z)=a_{\nu}^{(3)} \int_{\Omega} e^{-(z \mid u)} \psi(u) \Delta(u)^{\nu-\frac{N}{n}} m(d u),
$$

is an isometric isomorphism from the space $L_{\nu}^{2}(\Omega)$ of measurable functions $\psi$ on $\Omega$ such that

$$
\|\psi\|_{\nu}^{2}=a_{\nu}^{(3)} \int_{\Omega}|\psi(u)|^{2} \Delta(u)^{\nu-\frac{N}{n}} m(d u)<\infty,
$$

onto $\mathcal{H}_{\nu}^{2}\left(T_{\Omega}\right)$. The constant $a_{\nu}^{(3)}$ is such that the function $\Psi_{0}(u)=e^{-\operatorname{tr}(u)}$ has norm 1 , and then $\mathcal{L}_{\nu} \Psi_{0}=F_{0}$. Let us recall the definition of the multivariate Laguerre polynomials. The generalized binomial coefficients $\left(\begin{array}{c}\mathbf{m} \\ \mathbf{k}\end{array}\right)$ are defined by the generalized binomial formula:

$$
\Phi_{\mathbf{m}}(e+x)=\sum_{\mathbf{k} \subset \mathbf{m}}\left(\begin{array}{c}
\mathbf{m} \\
\mathbf{k}
\end{array}\right) \Phi_{\mathbf{k}}(x) .
$$

(Observe that $\left(\begin{array}{c}\mathbf{m} \\ \mathbf{k}\end{array}\right)$, as a function of $\mathbf{m}$, is a shifted Jack polynomial. With the notation of [Okunkov-Olshanski,1997],

$$
\left(\begin{array}{c}
\mathbf{m} \\
\mathbf{k}
\end{array}\right)=\frac{P_{\mathbf{k}}^{*}(\mathbf{m} ; \theta)}{H(\mathbf{k})}, \quad \theta=\frac{d}{2}
$$

see formula (3.8).) We define the multivariate Laguerre polynomial $\mathbf{L}_{\mathbf{m}}^{(\nu-1)}$ by

$$
\mathbf{L}_{\mathbf{m}}^{(\nu-1)}(x)=\frac{(\nu)_{\mathbf{m}}}{\left(\frac{N}{n}\right)_{\mathbf{m}}} \sum_{\mathbf{k} \subset \mathbf{m}}\left(\begin{array}{c}
\mathbf{m} \\
\mathbf{k}
\end{array}\right) \frac{1}{(\nu)_{\mathbf{k}}} \Phi_{\mathbf{k}}(-x) .
$$

(This definition slightly differs from the one given in [Faraut-Korányi,1994], p.343), but it has the advantage that it extends exactly the one variable case.) Define the Laguerre functions as $\Psi_{\mathbf{m}}^{(\nu)}(u)=e^{-\operatorname{tr}(u)} L_{\mathbf{m}}^{(\nu-1)}(2 u)$. Then

$$
\left(\mathcal{L}_{\nu} \Psi_{\mathbf{m}}^{(\nu)}\right)(z)=\frac{(\nu)_{\mathbf{m}}}{\left(\frac{N}{n}\right)_{\mathbf{m}}} F_{\mathbf{m}}^{(\nu)}(z) .
$$

([Faraut-Korányi,1994], Proposition XV.4.2.) The Laguerre functions form an orthogonal basis of $L_{\nu}^{2}(\Omega)^{K}$ of $K$-invariant functions in $L_{\nu}^{2}(\Omega)$.

Finally we perform a spherical Fourier transform which can be seen as a multivariate Mellin transform. Recall that the spherical function $\varphi_{\mathbf{s}}$, for $\mathbf{s} \in \mathbb{C}^{n}$, is defined on $\Omega$ by

$$
\varphi_{\mathbf{s}}(u)=\int_{K} \Delta_{\mathbf{s}+\rho}(k u) d k,
$$


where $\rho=\left(\rho_{1}, \ldots, \rho_{n}\right), \rho_{j}=\frac{d}{4}(2 j-n-1)$. ( $d k$ denotes the normalized Haar measure on the compact group $K$.) We introduce now the modified spherical Fourier transform $\mathcal{F}_{\nu}$ as follows: for a function $\psi$ on $\Omega$,

$$
\left(\mathcal{F}_{\nu} \psi\right)(\mathbf{s})=\frac{1}{\Gamma_{\Omega}\left(\mathbf{s}+\frac{\nu}{2}\right)} \int_{\Omega} \psi(u) \varphi_{\mathbf{s}}(u) \Delta(u)^{\frac{\nu}{2}-\frac{N}{n}} m(d u) .
$$

Observe that $\mathcal{F}_{\nu} \Psi_{0} \equiv 1$. By the spherical Plancherel theorem, $\mathcal{F}_{\nu}$ is an isometric isomorphism from $L_{\nu}^{2}(\Omega)^{K}$ onto the space $L^{2}\left(\mathbb{R}^{n}, M(d \lambda)\right)^{\mathfrak{S}_{n}}$ of square integrable symmetric functions on $\mathbb{R}^{n}$ with respect to the measure

$$
M(d \lambda)=a_{\nu}^{(4)}\left|\Gamma_{\Omega}\left(i \lambda+\rho+\frac{\nu}{2}\right)\right|^{2} \frac{1}{|c(i \lambda)|^{2}} m(d \lambda),
$$

where $c$ is the Harish-Chandra $c$-function and $a_{\nu}^{(4)}$ is such that $M(d \lambda)$ has total measure 1:

$$
\int_{\mathbb{R}^{n}}\left|\mathcal{F}_{\nu} \psi(i \lambda)\right|^{2} M(d \lambda)=a_{\nu}^{(3)} \int_{\Omega}|\psi(u)|^{2} \Delta(u)^{\nu-\frac{N}{n}} m(d u)
$$

The symbof $\sigma_{D}$ of a partial differential operator $D$ on $V$ is defined by

$$
D e^{(x \mid \xi)}=\sigma_{D}(x, \xi) e^{(x \mid \xi)} \quad(x, \xi \in V)
$$

( $D$ acts on the variable $x$.) If $D \in \mathbb{D}(\Omega)$, the algebra of $G$-invariant differential operators on $\Omega \simeq G / K$, then $\sigma_{D}$ is a $G$-invariant polynomial on $V \times V$ in the following sense: for $g \in G, \sigma_{D}(g x, \xi)=\sigma_{D}\left(x, g^{*} \xi\right)$. The map $D \mapsto \sigma_{D}(x, e)$ is a vector space isomorphism from $\mathbb{D}(\Omega)$ onto the space $\mathcal{P}(V)^{K}$ of $K$-invariant polynomials on $V$. The spherical function $\varphi_{\mathbf{s}}$ is an eigenfunction of every $D \in \mathbb{D}(\Omega): D \varphi_{\mathbf{s}}=\gamma_{D}(\mathbf{s}) \varphi_{\mathbf{s}}$, and the map $D \mapsto \gamma_{D}$ is an algebra isomorphism from $\mathbb{D}(\Omega)$ onto the algebra $\mathcal{P}\left(\mathbb{C}^{n}\right)^{\mathfrak{S}_{n}}$ of symmetric polynomials on $\mathbb{C}^{n}$.

In particular, let $D^{\mathbf{m}}$ denote the invariant differential operator $D$ for which $\sigma_{D}(x, e)=$ $\Phi_{\mathbf{m}}(x)$, and let $\gamma_{\mathbf{m}}=\gamma_{D^{\mathbf{m}}}$. Observe that, by the spherical Taylor formula ([FarautKorányi,1994], p.244),

$$
\left(\begin{array}{c}
\mathbf{m} \\
\mathbf{k}
\end{array}\right)=\frac{d_{\mathbf{k}}}{\left(\frac{N}{n}\right)_{\mathbf{k}}} \gamma_{\mathbf{k}}(\mathbf{m}-\rho)
$$

Lemma 2.1. - Let $\psi(u)=e^{-\operatorname{tr}(u)} p(u)$, where $p$ is a $K$-invariant polynomial on $V$. There is $D \in \mathbb{D}(\Omega)$ such that $\sigma_{D}(x,-e)=p(x)$. Then $\psi(u)=D e^{-\operatorname{tr}(u)}$, and the modified spherical transform of $\psi$ is given by

$$
\mathcal{F}_{\nu} \psi(\mathbf{s})=\gamma_{D}\left(-\mathbf{s}-\frac{\nu}{2}\right)
$$

Hence $q=\mathcal{F}_{\nu} \psi$ is a symmetric polynomial on $\mathbb{C}^{n}$. 
Proof. First part of the statement follows from what was explained above. Let us perform an integration by part as follows:

$$
\begin{aligned}
& \int_{\Omega} e^{-\operatorname{tr}(u)} p(u) \varphi_{\mathbf{s}}(u) \Delta(u)^{\frac{\nu}{2}-\frac{N}{n}} m(d u) \\
& =\int_{\Omega} D\left(e^{-\operatorname{tr}(u)}\right) \varphi_{\mathbf{s}+\frac{\nu}{2}}(u) \Delta(u)^{-\frac{N}{n}} m(d u) \\
& =\int_{\Omega} e^{-\operatorname{tr}(u)}\left(D^{*} \varphi_{\mathbf{s}+\frac{\nu}{2}}\right)(u) \Delta(u)^{-\frac{N}{n}} m(d u) \\
& =\gamma_{D^{*}}\left(\mathbf{s}+\frac{\nu}{2}\right) \int_{\Omega} e^{-\operatorname{tr}(u)} \varphi_{\mathbf{s}+\frac{\nu}{2}}(u) \Delta(u)^{-\frac{N}{n}} m(d u) \\
& =\gamma_{D^{*}}\left(\mathbf{s}+\frac{\nu}{2}\right) \Gamma_{\Omega}\left(\mathbf{s}+\frac{\nu}{2}\right)
\end{aligned}
$$

and recall that $\gamma_{D^{*}}(\mathbf{s})=\gamma_{D}(-\mathbf{s})$ ([Faraut-Korányi,1994], Proposition XIV.1.8).

We define the multivariate Meixner-Pollaczek polynomial $Q_{\mathbf{m}}^{(\nu)}(\mathbf{s})$ as the modified spherical Fourier transform of the Laguerre function $\Psi_{\mathbf{m}}^{(\nu)}$ :

$$
Q_{\mathbf{m}}^{(\nu)}(\mathbf{s})=\mathcal{F}_{\nu} \Psi_{\mathbf{m}}^{(\nu)}(\mathbf{s})
$$

Observing that, by Lemma 2.1,

$$
\mathcal{F}_{\nu}\left(e^{-\operatorname{tr} u} \Phi_{\mathbf{k}}\right)(\mathbf{s})=(-1)^{|\mathbf{k}|} \gamma_{\mathbf{k}}\left(-\mathbf{s}-\frac{\nu}{2}\right),
$$

we obtain:

Theorem 2.2. - The multivariate Meixner-Pollaczek polynomials $Q_{\mathbf{m}}^{(\nu)}$ form an orthogonal basis of the space $L^{2}\left(\mathbb{R}^{n}, M(d \lambda)\right)^{\mathfrak{S}_{n}}$. Furthermore

$$
Q_{\mathbf{m}}^{(\nu)}(\mathbf{s})=\frac{(\nu)_{\mathbf{m}}}{\left(\frac{N}{n}\right)_{\mathbf{m}}} \sum_{\mathbf{k} \subset \mathbf{m}} 2^{|\mathbf{k}|} \frac{1}{(\nu)_{\mathbf{k}}}\left(\begin{array}{c}
\mathbf{m} \\
\mathbf{k}
\end{array}\right) \gamma_{\mathbf{k}}\left(-\mathbf{s}-\frac{\nu}{2}\right) .
$$

Remark. In the one variable case $(V=\mathbb{R}), \gamma_{k}(s)=[s]_{k}$, and $\gamma_{k}\left(-s-\frac{\nu}{2}\right)=(-1)^{k}\left(s+\frac{\nu}{2}\right)_{k}$.

3. Generating formulae. - Following the same scheme as in Section 1, we will obtain the following generating formula for the multivariate Meixner-Pollaczek polynomials:

Theorem 3.1. - For $w \in \mathcal{D}, \nu \in \mathbb{C}, \mathbf{s} \in \mathbb{C}^{n}$,

$$
\sum_{\mathbf{m}} d_{\mathbf{m}} Q_{\mathbf{m}}^{(\nu)}(\mathbf{s}) \Phi_{\mathbf{m}}(w)=\Delta\left(e-w^{2}\right)^{-\frac{\nu}{2}} \varphi_{\mathbf{s}}\left((e-w)(e+w)^{-1}\right) .
$$

As in the one variable case, it follows that $Q_{\mathbf{m}}^{(\nu)}(-\mathbf{s})=(-1)^{|\mathbf{m}|} Q_{\mathbf{m}}^{(\nu)}(\mathbf{s})$. In [DavidsonÓlafsson-Zhang,2003], the polynomials $p_{\nu, \mathbf{m}}$ are defined through this generating formula. The definition on p. 179 is slightly different:

$$
d_{\mathbf{m}} Q_{\mathbf{m}}^{(\nu)}(\mathbf{s})=p_{\nu, \mathbf{m}}(i \mathbf{s}) .
$$


Proof. The reproducing kernel $\mathcal{K}_{\nu}$ for the weighted Bergman space $\mathcal{H}_{\nu}^{2}(D)$ is given by

$$
\mathcal{K}_{\nu}\left(w, w^{\prime}\right)=h\left(w, w^{\prime}\right)^{-\nu}=\sum_{\mathbf{m}}(\nu)_{\mathbf{m}} K^{\mathbf{m}}\left(w, w^{\prime}\right) .
$$

We consider its average over the compact group $K$, letting $\overline{w^{\prime}}=\zeta$ :

$$
H_{\nu}^{(1)}(w, \zeta)=\int_{K} h(w, k \bar{\zeta})^{-\nu} d k
$$

It is holomorphic in $w$ and $\zeta$. Then we obtain

$$
H_{\nu}^{(1)}(w, \zeta)=\sum_{\mathbf{m}} d_{\mathbf{m}} \frac{(\nu)_{\mathbf{m}}}{\left(\frac{N}{n}\right)_{\mathbf{m}}} \Phi_{\mathbf{m}}(\zeta) \Phi_{\mathbf{m}}(w) .
$$

We apply the transform $C_{\nu}$ to both handsides with respect to $\zeta$. The left handside becomes

$$
H_{\nu}^{(2)}(w, z)=2^{n \nu} \Delta(e-w)^{-\nu} \int_{K} \Delta(k z+c(w))^{-\nu} d k .
$$

We have used the identity $\Delta(z+e) h\left(w, c^{-1}(z)\right)=\Delta(e-w) \Delta(z+c(w))$. Since $C_{\nu} \Phi_{\mathbf{m}}=$ $F_{\mathbf{m}}^{(\nu)}$, we obtain

$$
H_{\nu}^{(2)}(w, z)=\sum_{\mathbf{m}} d_{\mathbf{m}} \frac{(\nu)_{\mathbf{m}}}{\left(\frac{N}{n}\right)_{\mathbf{m}}} \Phi_{\mathbf{m}}(w) F_{\mathbf{m}}^{(\nu)}(z)
$$

Next we apply the inverse of the modified Laplace transform $\mathcal{L}_{\nu}$ with respect to $z$. Define, for $w \in D, u \in \Omega$,

$$
H_{\nu}^{(3)}(w, u)=\Delta(e-w)^{-\nu} \int_{K} e^{-(k u \mid c(w))} d k .
$$

The modified Laplace transform of $H_{\nu}^{(3)}(w, u)$ with respect to $u$ is equal to $H_{\nu}^{(2)}(w, z)$. In fact

$$
\begin{aligned}
& \frac{2^{n \nu}}{\Gamma_{\Omega}(\nu)} \int_{\Omega} e^{-(z \mid u)} H_{\nu}^{(3)}(w, u) \Delta(u)^{\nu-\frac{N}{n}} m(d u) \\
& =\frac{2^{n \nu}}{\Gamma_{\Omega}(\nu)} \Delta(e-w)^{-\nu} \int_{\Omega}\left(\int_{K} e^{-(u \mid k z)} e^{-(u \mid c(w))} d k\right) \Delta(u)^{\nu-\frac{N}{n}} m(d u) \\
& =2^{n \nu} \Delta(e-w)^{-\nu} \int_{K} \Delta(k z+c(w))^{-\nu} d k=H_{\nu}^{(2)}(w, z) .
\end{aligned}
$$

On the other hand, since:

$$
\mathcal{L}_{\nu}\left(\Psi_{\mathbf{m}}^{(\nu)}\right)=\frac{(\nu)_{\mathbf{m}}}{\left(\frac{N}{n}\right)_{\mathbf{m}}} F_{m}^{(\nu)},
$$

we get the generating formula for the Laguerre functions: 
Proposition 3.2.

$$
\sum_{\mathbf{m}} d_{\mathbf{m}} \Psi_{\mathbf{m}}^{(\nu)}(u) \Phi_{\mathbf{m}}(w)=H_{\nu}^{(3)}(w, u)
$$

Finally we perform the modified spherical Fourier transform $\mathcal{F}_{\nu}$ with respect to $u$. Define, for $w \in D, \mathbf{s} \in \mathbb{C}^{r}$,

$$
\begin{aligned}
H_{\nu}^{(4)}(w, \mathbf{s}) & =\Delta\left(e-w^{2}\right)^{-\frac{\nu}{2}} \varphi_{\mathbf{s}}\left((e-w)(e+w)^{-1}\right) \\
& =\Delta\left(e-w^{2}\right)^{-\frac{\nu}{2}} \varphi_{-\mathbf{s}}(c(w))
\end{aligned}
$$

since $\varphi_{\mathbf{s}}\left(x^{-1}\right)=\varphi_{-\mathbf{s}}(x)$. The modified spherical Fourier transform of $H_{\nu}^{(3)}(w, u)$ with respect to $u$ is equal to $H_{\nu}^{(4)}(w, \mathbf{s})$. In fact

$$
\begin{aligned}
& \frac{1}{\Gamma_{\Omega}\left(\mathbf{s}+\frac{\nu}{2}\right)} \int_{\Omega} H_{\nu}^{(3)}(w, u) \varphi_{\mathbf{s}}(u) \Delta(u)^{\frac{\nu}{2}-\frac{N}{n}} m(d u) \\
& =\frac{1}{\Gamma_{\Omega}\left(\mathbf{s}+\frac{\nu}{2}\right)} \Delta(e-w)^{-\nu} \int_{\Omega} e^{-\left(u \mid(e+w)(e-w)^{-1}\right)} \varphi_{\mathbf{s}}(u) \Delta(u)^{\frac{\nu}{2}-\frac{N}{n}} m(d u) \\
& =\Delta(e-w)^{-\nu} \varphi_{\mathbf{s}+\frac{\nu}{2}}\left((e-w)(e+w)^{-1}\right) \\
& =\Delta\left(e-w^{2}\right)^{-\frac{\nu}{2}} \varphi_{\mathbf{s}}\left((e-w)(e+w)^{-1}\right) .
\end{aligned}
$$

By definition the Meixner-Pollaczek polynomial $Q_{\mathbf{m}}^{(\nu)}$ is the modified spherical Fourier transform of the Laguerre function $\Psi_{\mathbf{m}}^{(\nu)}$. Hence:

$$
\sum_{\mathbf{m}} d_{\mathbf{m}} Q_{m}^{(\nu)}(\mathbf{s}) \Phi_{\mathbf{m}}(w)=\Delta\left(e-w^{2}\right)^{-\frac{\nu}{2}} \varphi_{\mathbf{s}}\left((e-w)(e+w)^{-1}\right)
$$

4. Determinantal formulae. - In the case $d=2(V=\operatorname{Herm}(n, \mathbb{C}), K=U(n))$, there are determinantal formulae for the Laguerre functions $\Psi_{\mathbf{m}}^{(\nu)}$ and for the MeixnerPollaczek polynomials $Q_{\mathbf{m}}^{(\nu)}$. Consider a Jordan frame $\left\{c_{1}, \ldots, c_{n}\right\}$ in $V$, and let $\delta=$ $(n-1, n-2, \ldots, 1,0)$.

THEOREM 4.1. - Assume $d=2$. The multivariate Laguerre function $\Psi_{\mathbf{m}}^{(\nu)}$ admits the following determinantal formula involving the one variable Laguerre functions $\psi_{m}^{(\nu)}$ : for $u=\sum_{j=1}^{n} u_{i} c_{i}$

$$
\Psi_{\mathbf{m}}^{(\nu)}(u)=\delta ! 2^{-\frac{1}{2} n(n-1)} \frac{\operatorname{det}\left(\psi_{m_{j}+\delta_{j}}^{(\nu-n+1)}\left(u_{i}\right)\right)_{1 \leq i, j \leq n}}{V\left(u_{1}, \ldots, u_{n}\right)},
$$

where $V$ denote the Vandermonde polynomial:

$$
V\left(u_{1}, \ldots, u_{n}\right)=\prod_{i<j}\left(u_{j}-u_{i}\right)
$$


As a result one obtains the following determinantal formula for the multivariate Laguerre polynomials:

$$
\mathbf{L}_{\mathbf{m}}^{\nu}(u)=\delta ! \frac{\operatorname{det}\left(L_{m_{j}+\delta_{j}}^{(\nu-n+1)}\left(u_{i}\right)\right)_{1 \leq i, j \leq n}}{V\left(u_{1}, \ldots, u_{n}\right)} .
$$

Proof. We start from the generating function for the Laguerre functions $\Psi_{\mathbf{m}}^{(\nu)}$ (Proposition $3.2)$ :

$$
\begin{aligned}
H_{\nu}^{(3)}(u, w) & =\Delta(e-w)^{-\nu} \int_{K} e^{-\left(k u \mid(e+w)(e-w)^{-1}\right)} d k \\
& =\sum_{\mathbf{m}} d_{\mathbf{m}} \Phi_{\mathbf{m}}(w) \Psi_{\mathbf{m}}^{(\nu)}(u) .
\end{aligned}
$$

In the case $d=2$, the evaluation of the following integral is classical: for $x=\sum_{i=1}^{n} x_{i} c_{i}$, $y=\sum_{j=1}^{n} y_{j} c_{j}$, then

$$
\mathcal{I}(x, y)=\int_{K} e^{(k x \mid y)} d k=\delta ! \frac{\operatorname{det}\left(e^{x_{i} y_{j}}\right)}{V\left(x_{1}, \ldots, x_{n}\right) V\left(y_{1}, \ldots, y_{n}\right)} .
$$

In fact $\mathcal{I}(x, y)$ is the so called Itzykson-Zuber integral:

$$
\mathcal{I}(x, y)=\int_{U(n)} e^{\operatorname{tr}\left(u x u^{*} y\right)} d u .
$$

Therefore, for $u=\sum_{i=1}^{n} u_{i} c_{i}, w=\sum_{j=1}^{n} w_{j} c_{j}$,

$$
H_{\nu}^{(3)}(u, w)=\delta ! \prod_{j=1}^{n}\left(1-w_{j}\right)^{-\nu} \frac{\operatorname{det}\left(e^{-u_{i} \frac{1+w_{j}}{1-w_{j}}}\right)}{V\left(u_{1}, \ldots, u_{n}\right) V\left(\frac{1+w_{1}}{1-w_{1}}, \ldots, \frac{1+w_{n}}{1-w_{n}}\right)} .
$$

Noticing that

$$
\frac{1+w_{j}}{1-w_{j}}-\frac{1+w_{k}}{1-w_{k}}=2 \frac{w_{j}-w_{k}}{\left(1+w_{j}\right)\left(1+w_{k}\right)}
$$

we obtain

$$
H_{\nu}^{(3)}(u, w)=\delta ! 2^{-\frac{1}{2} n(n-1)} \frac{\operatorname{det}\left(\left(1-w_{j}\right)^{-(\nu-n+1)} e^{-u_{i} \frac{1+w_{j}}{1-w_{j}}}\right)}{V\left(u_{1}, \ldots, u_{n}\right) V\left(w_{1}, \ldots, w_{n}\right)} .
$$

We will expand the above expression in Schur function series by using a formula due to Hua.

Lemma 4.2. - Consider $n$ power series

$$
f_{i}(w)=\sum_{m=0}^{\infty} c_{m}^{(i)} w^{m} \quad(i=1, \ldots, n)
$$


Then

$$
\frac{\operatorname{det}\left(f_{i}\left(w_{j}\right)\right)}{V\left(w_{1}, \ldots, w_{n}\right)}=\sum_{\mathbf{m}} a_{\mathbf{m}} s_{\mathbf{m}}\left(w_{1}, \ldots, w_{n}\right),
$$

where $s_{\mathbf{m}}$ is the Schur function associated to the partition $\mathbf{m}$, and

$$
a_{\mathbf{m}}=\operatorname{det}\left(c_{m_{j}+\delta_{j}}^{(i)}\right) .
$$

(See [Hua,1963], Theorem 1.2.1, p.22).

Let $\nu^{\prime}=\nu-n+1$, and consider the $n$ power series

$$
f_{i}(w)=(1-w)^{-\nu^{\prime}} e^{-u_{i} \frac{1+w}{1-w}}=\sum_{m=0}^{\infty} \psi_{m}^{\left(\nu^{\prime}\right)}\left(u_{i}\right) w^{m} .
$$

Since

$$
d_{\mathbf{m}} \Phi_{\mathbf{m}}\left(\sum_{j=1}^{n} w_{j} c_{j}\right)=s_{\mathbf{m}}\left(w_{1}, \ldots, w_{n}\right)
$$

we obtain

$$
\Psi_{\mathbf{m}}^{(\nu)}(u)=\delta ! 2^{-\frac{1}{2} n(n-1)} \frac{\operatorname{det}\left(\psi_{m_{j}+\delta_{j}}^{(\nu-n+1)}\left(u_{i}\right)\right)}{V\left(u_{1}, \ldots, u_{n}\right)}
$$

By using the same method we will obtain a determinantal formula for the MeixnerPollaczek polynomials $Q_{\mathbf{m}}^{(\nu)}$

THEOREM 4.3.

$$
Q_{\mathbf{m}}^{(\nu)}(\mathbf{s})=\delta !(-2)^{-\frac{1}{2} n(n-1)} \frac{\operatorname{det}\left(q_{m_{j}+\delta_{j}}^{\nu-n+1}\left(s_{i}\right)\right)_{1 \leq i, j \leq n}}{V\left(s_{1}, \ldots, s_{n}\right)},
$$

where $q_{m}^{(\nu)}$ denote the one variable Meixner-Pollaczek polynomials.

Proof. We start from the generating formula for the Meixner-Pollaczek polynomials $Q_{\mathbf{m}}^{(\nu)}$ (Theorem 3.1):

$$
\Delta\left(e-w^{2}\right)^{-\frac{\nu}{2}} \varphi_{\mathbf{s}}\left((e-w)(e+w)^{-1}\right)=\sum_{\mathbf{m}} d_{\mathbf{m}} Q_{\mathbf{m}}^{(\nu)}(\mathbf{s}) \Phi_{\mathbf{m}}(w) .
$$

For $x=\sum_{i=1}^{n} x_{i} c_{i}$, the spherical function $\varphi_{\mathbf{s}}(x)$ is essentially a Schur function in the variables $x_{1}, \ldots, x_{n}$ :

$$
\varphi_{\mathbf{s}}(x)=\delta !\left(x_{1} x_{2} \ldots x_{r}\right)^{\frac{1}{2}(n-1)} \frac{\operatorname{det}\left(x_{j}^{s_{i}}\right)}{V\left(s_{1}, \ldots, s_{n}\right) V\left(x_{1}, \ldots, x_{n}\right)} .
$$


Let us compute now, for $w=\sum_{j=1}^{n} w_{j} c_{j}$,

$$
\begin{aligned}
& \Delta\left(e-w^{2}\right)^{-\frac{\nu}{2}} \varphi_{\mathbf{s}}\left((e-w)(e+w)^{-1}\right) \\
& =\delta ! \prod_{j=1}^{n}\left(1-w_{j}^{2}\right)^{-\frac{\nu}{2}} \prod_{j=1}^{n}\left(\frac{1-w_{j}}{1+w_{j}}\right)^{\frac{1}{2}(n-1)} \frac{\operatorname{det}\left(\left(\frac{1-w_{j}}{1+w_{j}}\right)^{s_{i}}\right)}{V\left(s_{1}, \ldots, s_{n}\right) V\left(\frac{1-w_{1}}{1+w_{1}}, \ldots, \frac{1-w_{n}}{1+w_{n}}\right)} .
\end{aligned}
$$

In the same way as for the proof of Theorem 4.1, we obtain

$$
\begin{aligned}
& \Delta\left(e-w^{2}\right)^{-\frac{\nu}{2}} \varphi_{\mathbf{s}}\left((e-w)(e+w)^{-1}\right) \\
& =\delta !(-2)^{-\frac{1}{2} n(n-1)} \frac{\operatorname{det}\left(\left(1-w_{j}\right)^{s_{i}-\frac{\nu}{2}+\frac{1}{2}(n-1)}\left(1+w_{j}\right)^{-s_{i}-\frac{\nu}{2}+\frac{1}{2}(n-1)}\right)}{V\left(s_{1}, \ldots, s_{n}\right) V\left(w_{1}, \ldots, w_{n}\right)} .
\end{aligned}
$$

We apply once more Lemma 4.2 to the $n$ power series

$$
f_{i}(w)=(1-w)^{s_{i}-\frac{\nu^{\prime}}{2}}(1+w)^{-s_{i}-\frac{\nu^{\prime}}{2}}=\sum_{m}^{\infty} q_{m}^{\nu^{\prime}}\left(s_{i}\right) w^{m}
$$

with $\nu^{\prime}=\nu-n+1$, and obtain finally:

$$
Q_{\mathbf{m}}^{(\nu)}(\mathbf{s})=\delta !(-2)^{-\frac{1}{2} n(n-1)} \frac{\operatorname{det}\left(q_{m_{j}+\delta_{j}}^{\nu-n+1}\left(s_{i}\right)\right)}{V\left(s_{1}, \ldots, s_{n}\right)} .
$$

5. Difference equation and Pieri's formula for the multivariate MeixnerPollaczek polynomials. — Let us first recall Pieri's formula for the spherical functions.

Proposition 5.1.

$$
(\operatorname{tr} x) \varphi_{\mathbf{s}}(x)=\sum_{j=1}^{n} \alpha_{j}(\mathbf{s}) \varphi_{\mathbf{s}+\varepsilon_{j}}(x)
$$

with

$$
\alpha_{j}(\mathbf{s})=\prod_{k \neq j} \frac{s_{j}-s_{k}+\frac{d}{2}}{s_{j}-s_{k}} .
$$

$\left(\left\{\varepsilon_{j}\right\}\right.$ denote the canonical basis of $\left.\mathbb{C}^{n}.\right)$

See [Dib, 1990], Proposition 6.1 (with a minor correction), where it is called Kushner's formula. (See also [Zhang,1995], Theorem 1). One observes that

$$
\alpha_{j}(\mathbf{s})=\frac{c(\mathbf{s})}{c\left(\mathbf{s}+\varepsilon_{j}\right)}
$$


in agreement with the asymptotic behaviour of the spherical function $\varphi_{\mathbf{s}}$ :

for $\mathbf{s}=\left(s_{1}, \ldots, s_{n}\right)$ real, with $s_{1}<\ldots<s_{n}$, and $a=\sum_{i=1}^{n} a_{i} c_{i}$ with $a_{1}<\ldots<a_{r}$,

$$
\varphi_{\mathbf{s}}(\exp t a) \sim c(\mathbf{s}) e^{(\rho+\mathbf{s} \mid a) t} \quad(t \rightarrow \infty) .
$$

Recall that the $c$-function is a product of beta functions:

$$
c(\mathbf{s})=c_{0} \prod_{j<k} B\left(s_{j}-s_{k}, \frac{d}{2}\right) .
$$
obtains

By puting $\mathbf{m}=\mathbf{s}+\rho$ (recall that $\left.\rho_{j}=\frac{d}{4}(2 j-n-1)\right), s_{j}=m_{j}-\frac{d}{2} j+\frac{d}{4}(n+1)$, one

$$
(\operatorname{tr} x) \Phi_{\mathbf{m}}(x)=\sum_{j=1}^{n} a_{j}(\mathbf{m}) \Phi_{\mathbf{m}+\varepsilon_{j}}(x),
$$

with

$$
a_{j}(\mathbf{m})=\prod_{k \neq j} \frac{m_{j}-m_{k}-\frac{d}{2}(j-k-1)}{m_{j}-m_{k}-\frac{d}{2}(j-k)} .
$$

(In agreement with Lassalle's results [1998], p.320, 1.-4.)

We introduce the difference operator acting on functions on $\mathbb{C}^{n}$ :

$$
\begin{aligned}
\left(D_{\nu} f\right)(\mathbf{s}) & =\sum_{j=1}^{n}\left(s_{j}+\frac{\nu}{2}-\frac{d}{4}(n-1)\right) \alpha_{j}(\mathbf{s}) f\left(\mathbf{s}+\varepsilon_{j}\right) \\
& +\sum_{j=1}^{n}\left(-s_{j}-\frac{\nu}{2}+\frac{d}{4}(n-1)\right) \alpha_{j}(-\mathbf{s}) f\left(\mathbf{s}-\varepsilon_{j}\right) .
\end{aligned}
$$

Theorem 5.2. - (i) The Meixner-Pollaczek polynomials $Q_{\mathbf{m}}^{(\nu)}$ are eigenfunctions of the operator $D_{\nu}$ :

$$
D_{\nu} Q_{\mathbf{m}}^{(\nu)}=(2|\mathbf{m}|+\nu) Q_{\mathbf{m}}^{(\nu)}
$$

(ii) Furthermore they satisfy the following Pieri's formula:

$$
\begin{aligned}
& \left(2 \sum_{j=1}^{n} s_{j}\right) d_{\mathbf{m}} Q_{\mathbf{m}}^{(\nu)}(\mathbf{s}) \\
& =\sum_{j=1}^{n}\left(m_{j}+\nu-1-\frac{d}{2}(j-1)\right) \alpha_{j}\left(\mathbf{m}-\varepsilon_{j}-\rho\right) d_{\mathbf{m}-\varepsilon_{j}} Q_{\mathbf{m}-\varepsilon_{j}}^{(\nu)}(\mathbf{s}) \\
& -\sum_{j=1}^{n}\left(m_{j}+1+\frac{d}{2}(n-j)\right) \alpha_{j}\left(-\mathbf{m}-\varepsilon_{j}+\rho\right) d_{\mathbf{m}+\varepsilon_{j}} Q_{\mathbf{m}+\varepsilon_{j}}^{(\nu)}(\mathbf{s}) .
\end{aligned}
$$

Statement (i) has been obtained in another way in [Davidson,Ólafsson-Zhang,2003]: Theorem 6.1. 
Proof. (1) We consider first the differential operator $D_{\nu}^{(1)}$ on the bounded domain $\mathcal{D}$ as

$$
D_{\nu}^{(1)} f(w)=2\langle w, \nabla f(w)\rangle+n \nu f(w)
$$

This is a modified Euler operator. (We denote by $\langle z, w\rangle$ the $\mathbb{C}$-bilinear form on $V_{\mathbb{C}}$ extending the Euclidean inner product on $V$ so that $(z \mid w)=\langle z, \bar{w}\rangle$.) The functions $\Phi_{\mathbf{m}}$, being homogeneous, are eigenfunctions of $D_{\nu}^{(1)}$ :

$$
D_{\nu}^{(1)} \Phi_{\mathbf{m}}=(2|\mathbf{m}|+n \nu) \Phi_{\mathbf{m}}
$$

(2) Next we define the operator $D_{\nu}^{(2)}$ on the tube domain $T_{\Omega}$ as the image of $D_{\nu}^{(1)}$ through the Cayley transform. More precisely $D_{\nu}^{(2)} C_{\nu}=C_{\nu} D_{\nu}^{(1)}$.

Proposition 5.3.

$$
D_{\nu}^{(2)} F(z)=\left\langle z^{2}-e, \nabla f(z)\right\rangle+\nu \operatorname{tr}(z) F(z)
$$

This means that the symbol $\sigma_{\nu}^{(2)}$ of $D_{\nu}^{(2)}$ is equal to

$$
\sigma_{\nu}^{(2)}(z, \zeta)=\left\langle z^{2}-e, \zeta\right\rangle+\nu \operatorname{tr} z
$$

Proof. We will use the following formulae: $\nabla\left(\Delta(x)^{\alpha}\right)=\alpha \Delta(x)^{\alpha} x^{-1}$ (Proposition III.4.2 in [Faraut-Korányi,1994]), and, if $j$ denotes the inversion: $j(x)=x^{-1}$, then $(D j)_{x}=$ $-P\left(x^{-1}\right)$ ( $P$ is the so-called quadratic representation of the Jordan algebra $V$ ). By writing $c(w)=2(e-w)^{-1}-e$, we obtain the differential of the Cayley transform: $(D c)_{w}=$ $2 P\left((e-w)^{-1}\right)$. If $F=C_{\nu} f$, i.e. $f(w)=\Delta(e-w)^{-\nu} F(c(w))$, then

$$
\begin{aligned}
D_{\nu}^{(1)} f(w) & =2\langle\nabla f(w), w\rangle+n \nu f(w) \\
& =2 \nu\left\langle(e-w)^{-1}, w\right\rangle \Delta(e-w)^{-\nu} F(c(w)) \\
& +4 \Delta(e-w)^{-\nu}\left\langle\nabla F(c(w)), P\left((e-w)^{-1}\right) w\right\rangle+n \nu f(w) .
\end{aligned}
$$

With $z=c(w)$, one gets

$$
2\left\langle(e-w)^{-1}, w\right\rangle=\operatorname{tr}(z)-n, \quad 4 P\left((e-w)^{-1}\right) w=z^{2}-e .
$$

Therefore

$$
D_{\nu}^{(1)} f(w)=\Delta(e-w)^{-\nu} G(c(w))
$$

with

$$
G(z)=\left\langle z^{2}-e, \nabla F(z)\right\rangle+\nu \operatorname{tr}(z) F(z) .
$$

The functions $F_{\mathbf{m}}^{(\nu)}$ are eigenfunctions of $D_{\nu}^{(2)}$ :

$$
D_{\nu}^{(2)} F_{\mathbf{m}}^{(\nu)}=(2|\mathbf{m}|+n \nu) F_{\mathbf{m}}^{(\nu)} .
$$


(3) Now the differential operator $D_{\nu}^{(3)}$ is defined on $\Omega$ as the inverse image of $D_{\nu}^{(2)}$ under the modified Laplace transform.

$$
\mathcal{L}_{\nu} \circ D_{\nu}^{(3)}=D_{\nu}^{(2)} \circ \mathcal{L}_{\nu}
$$

Proposition 5.4.

$$
D_{\nu}^{(3)}=-\left\langle u,\left(\frac{\partial}{\partial u}\right)^{2}\right\rangle-\nu \operatorname{tr}\left(\frac{\partial}{\partial u}\right)+\operatorname{tr}(u) .
$$

This means that $D_{\nu}^{(3)}$ is the differential operator with symbol

$$
\sigma_{\nu}^{(3)}(u, \xi)=-\left\langle u, \xi^{2}\right\rangle-\nu \operatorname{tr} \xi+\operatorname{tr}(u)
$$

Proof. By writing

$$
\left(\mathcal{L}_{\nu} \psi\right)(z)=\left(e^{-(z \mid u)} \mid \bar{\psi}\right)_{L_{\nu}^{2}(\Omega)}
$$

and observing that $D_{\nu}^{(2)}$ is formally selfadjoint, we obtain

$$
\mathcal{L}_{\nu}\left(D_{\nu}^{(3)} \psi\right)(z)=\left(D_{\nu}^{(3)} e^{-(z \mid u)} \mid \bar{\psi}\right)
$$

It follows that

$$
D_{\nu}^{(3)} e^{-(z \mid u)}=D_{\nu}^{(2)} e^{-(z \mid u)}
$$

(The operator $D_{\nu}^{(2)}$ acts on the variable $z$, while $D_{\nu}^{(3)}$ acts on the variable $u$.)

The Laguerre functions $\Psi_{\mathbf{m}}^{(\nu)}$ are eigenfunctions of $D_{\nu}^{(3)}$ :

$$
D_{\nu}^{(3)} \Psi_{\mathbf{m}}^{(\nu)}=(2|\mathbf{m}|+n \nu) \Psi_{\mathbf{m}}^{(\nu)} .
$$

(Actually the Laguerre functions are solutions of a system of differential equations and the above equation is one of them, see [Ricci-Vignati,1994].)

In order to determine the operator $D_{\nu}^{(4)}$ acting on functions on $\mathbb{C}^{n}$ such that $D_{\nu}^{(4)} \mathcal{F}_{\nu}=$ $\mathcal{F}_{\nu} D_{\nu}^{(3)}$ we need some preliminaries.

LEMMA 5.5.

$$
\operatorname{tr}\left(\nabla \varphi_{\mathbf{s}}(x)\right)=\sum_{j=1}^{n}\left(s_{j}+\frac{d}{4}(n-1)\right) \alpha_{j}(-s) \varphi_{\mathbf{s}-\varepsilon_{j}}(x) .
$$

(In agreement with Lassalle's results [1998], p.321, first line of (14.1).) 
Proof. For $t>0$ we consider the following Laplace integral:

$$
\int_{\Omega} e^{-(x \mid y)} e^{-t \operatorname{tr} y} \varphi_{\mathbf{s}}(y) \Delta(y)^{-\frac{N}{n}} m(d y)=\Gamma_{\Omega}(\mathbf{s}+\rho) \varphi_{-\mathbf{s}}(t e+x) .
$$

Taking the derivatives with respect to $\mathrm{t}$ for $t=0$, one gets:

$$
-\int_{\Omega} e^{-(x \mid y)} \operatorname{tr} y \varphi_{\mathbf{s}}(y) \Delta(y)^{-\frac{N}{n}} m(d y)=\Gamma_{\Omega}(\mathbf{s}+\rho) \operatorname{tr}\left(\nabla \varphi_{-\mathbf{s}}(x)\right) .
$$

By using Proposition 4.1:

$$
\operatorname{tr} y \varphi_{\mathbf{s}}(y)=\sum_{j=1}^{n} \alpha_{j}(\mathbf{s}) \varphi_{\mathbf{s}+\varepsilon_{j}}(y)
$$

and since

$$
\sum_{j=1}^{n} \alpha_{j}(\mathbf{s}) \int_{\Omega} e^{-(x \mid y)} \varphi_{\mathbf{s}+\varepsilon_{j}}(y) \Delta(y)^{-\frac{N}{n}} m(d y)=\sum_{j=1}^{n} \alpha_{j}(\mathbf{s}) \Gamma_{\Omega}\left(\mathbf{s}+\varepsilon_{j}+\rho\right) \varphi_{-\mathbf{s}-\varepsilon_{j}}(x),
$$

one obtains

$$
\begin{aligned}
\operatorname{tr}\left(\nabla \varphi_{-\mathbf{s}}(x)\right) & =-\sum_{j=1}^{n} \alpha_{j}(\mathbf{s}) \frac{\Gamma_{\Omega}\left(\mathbf{s}+\varepsilon_{j}+\rho\right)}{\Gamma_{\Omega}(\mathbf{s}+\rho)} \varphi_{-\mathbf{s}-\varepsilon_{j}}(x) \\
& =-\sum_{j=1}^{n} \alpha_{j}(\mathbf{s})\left(s_{j}-\frac{d}{4}(n-1)\right) \varphi_{-\mathbf{s}-\varepsilon_{j}}(x)
\end{aligned}
$$

or

$$
\operatorname{tr}\left(\nabla \varphi_{\mathbf{s}}(x)\right)=\sum_{j=1}^{n} \alpha_{j}(-\mathbf{s})\left(s_{j}+\frac{d}{4}(n-1)\right) \varphi_{\mathbf{s}-\varepsilon_{j}}(x) .
$$

In fact, by the explicit formula for $\Gamma_{\Omega}$,

$$
\Gamma_{\Omega}(\mathbf{s}+\rho)=(2 \pi)^{N-n} \prod_{j=1}^{n} \Gamma\left(s_{j}-\frac{d}{4}(n-1)\right),
$$

and

$$
\frac{\Gamma_{\Omega}\left(\mathbf{s}+\varepsilon_{j}+\rho\right)}{\Gamma_{\Omega}(\mathbf{s}+\rho)}=\frac{\Gamma\left(s_{j}+1-\frac{d}{4}(n-1)\right)}{\Gamma\left(s_{j}-\frac{d}{4}(n-1)\right)}=s_{j}-\frac{d}{4}(n-1) .
$$

LEMMA 5.6.

$$
\left\langle\nabla \varphi_{\mathbf{s}}(x), x^{2}\right\rangle=\sum_{j=1}^{r}\left(s_{j}-\frac{d}{4}(r-1)\right) \alpha_{j}(s) \varphi_{\mathbf{s}+\varepsilon_{j}}(x) .
$$


Proof. One uses the following formula: if $F(x)=f\left(x^{-1}\right)$, then

$$
\left\langle\nabla F(x), x^{2}\right\rangle=-\left\langle\nabla f\left(x^{-1}\right), P\left(x^{-1}\right) x^{2}\right\rangle=-\operatorname{tr}\left(\nabla f\left(x^{-1}\right)\right) .
$$

Taking $f(x)=\varphi_{-\mathbf{s}}(x), F(x)=\varphi_{\mathbf{s}}(x)$, one obtains:

$$
\begin{aligned}
\left\langle\nabla \varphi_{\mathbf{s}}(x), x^{2}\right\rangle & =-\operatorname{tr}\left(\nabla \varphi_{-\mathbf{s}}\left(x^{-1}\right)\right) \\
& =-\sum_{j=1}^{n}\left(-s_{j}+\frac{d}{4}(n-1)\right) \alpha_{j}(\mathbf{s}) \varphi_{-\mathbf{s}-\varepsilon_{j}}\left(x^{-1}\right) \\
& =\sum_{j=1}^{n}\left(s_{j}-\frac{d}{4}(n-1)\right) \alpha_{j}(\mathbf{s}) \varphi_{\mathbf{s}+\varepsilon_{j}}(x) .
\end{aligned}
$$

LEMMA 5.7.

$$
\begin{aligned}
D_{\nu}^{(2)} \varphi_{\mathbf{s}}(z) & =\sum_{j=1}^{n}\left(s_{j}+\nu-\frac{d}{4}(n-1)\right) \alpha_{j}(\mathbf{s}) \varphi_{\mathbf{s}+\varepsilon_{j}}(z) \\
& -\sum_{j=1}^{n}\left(s_{j}+\frac{d}{4}(n-1)\right) \alpha_{j}(-\mathbf{s}) \varphi_{\mathbf{s}-\varepsilon_{j}}(z) .
\end{aligned}
$$

For $\mathbf{s}=\mathbf{m}-\rho$, one obtains

$$
\begin{aligned}
\left(D_{\nu}^{(2)} \Phi_{\mathbf{m}}\right)(z) & =\sum_{j=1}^{n}\left(m_{j}+\nu-\frac{d}{2}(j-1)\right) \alpha_{j}(\mathbf{m}-\rho) \Phi_{\mathbf{m}+\varepsilon_{j}}(z) \\
& -\sum_{j=1}^{n}\left(m_{j}+\frac{d}{2}(n-j)\right) \alpha_{j}(-\mathbf{m}+\rho) \Phi_{\mathbf{m}-\varepsilon_{j}}(z) .
\end{aligned}
$$

(Theorem 2.2 in [Ørsted-Zhang,1995b] is an equivalent result.)

Proof. By Lemmas 5.5, 5.6, and Proposition 5.1, we obtain

$$
\begin{aligned}
& D_{\nu}^{(2)} \varphi_{\mathbf{s}}(z)=\left\langle z^{2}, \nabla \varphi_{\mathbf{s}}(z)\right\rangle-\left\langle e, \nabla \varphi_{\mathbf{s}}(z)\right\rangle+\nu(\operatorname{tr} z) \varphi_{\mathbf{s}}(z) \\
& =\sum_{j=1}^{n}\left(s_{j}-\frac{d}{4}(n-1)\right) \alpha_{j}(s) \varphi_{\mathbf{s}+\varepsilon_{j}}(z)-\sum_{j=1}^{n}\left(s_{j}+\frac{d}{4}(n-1)\right) \alpha_{j}(-\mathbf{s}) \varphi_{\mathbf{s}-\varepsilon_{j}}(z) \\
& +\nu \sum_{j=1}^{n} \alpha_{j}(\mathbf{s}) \varphi_{\mathbf{s}+\varepsilon_{j}}(z) \\
& =\sum_{j=1}^{n}\left(s_{j}+\nu-\frac{d}{4}(n-1)\right) \alpha_{j}(\mathbf{s}) \varphi_{\mathbf{s}+\varepsilon_{j}}(z)-\sum_{j=1}^{n}\left(s_{j}+\frac{d}{4}(n-1)\right) \alpha_{j}(-\mathbf{s}) \varphi_{\mathbf{s}-\varepsilon_{j}}(z) .
\end{aligned}
$$


Lemma 5.8.

$$
\begin{aligned}
& D_{\nu}^{(3)} \varphi_{\mathbf{s}}(u) \\
= & -\sum_{j=1}^{n}\left(s_{j}+\frac{d}{4}(n-1)\right)\left(s_{j}+\nu-1-\frac{d}{4}(n-1)\right) \alpha_{j}(-\mathbf{s}) \varphi_{\mathbf{s}-\varepsilon_{j}}(u) \\
& +\sum_{j=1}^{n} \alpha_{j}(\mathbf{s}) \varphi_{\mathbf{s}+\varepsilon_{j}}(u) .
\end{aligned}
$$

Proof. Recall that the operator $D_{\nu}^{(3)}$ is defined by the relation $\mathcal{L}_{\nu} D_{\nu}^{(3)}=D_{\nu}^{(2)} \mathcal{L}_{\nu}$. The modified Laplace transform of $\varphi_{\mathbf{s}}$ is given by:

$$
\begin{aligned}
\left(\mathcal{L}_{\nu} \varphi_{\mathbf{s}}\right)(z) & =\frac{2^{r \nu}}{\Gamma_{\Omega}(\nu)} \int_{\Omega} e^{-(z \mid u)} \varphi_{\mathbf{s}}(u) \Delta(u)^{\nu-\frac{n}{r}} d u \\
& =\frac{2^{r \nu}}{\Gamma_{\Omega}(\nu)} \Gamma_{\Omega}(\mathbf{s}+\nu+\rho) \varphi_{-\mathbf{s}-\nu}(z) .
\end{aligned}
$$

We obtain, by using Lemma 5.7,

$$
\begin{aligned}
D_{\nu}^{(2)}\left(\mathcal{L}_{\nu} \varphi_{\mathbf{s}}\right)(z) & =\frac{2^{r \nu}}{\Gamma_{\Omega}(\nu)} \Gamma_{\Omega}(\mathbf{s}+\nu+\rho) \times \\
& \left(-\sum_{j=1}^{n}\left(s_{j}+\frac{d}{4}(n-1)\right) \alpha_{j}(-\mathbf{s}-\nu) \varphi_{-\mathbf{s}-\nu+\varepsilon_{j}}(z)\right. \\
& \left.+\sum_{j=1}^{n}\left(s_{j}+\nu-\frac{d}{4}(n-1)\right) \alpha_{j}(\mathbf{s}+\nu) \varphi_{-\mathbf{s}-\nu-\varepsilon_{j}}(z)\right),
\end{aligned}
$$

and this can be written

$$
\begin{aligned}
& D_{\nu}^{(2)}\left(\mathcal{L}_{\nu} \varphi_{\mathbf{s}}\right)(z)= \\
& -\sum_{j=1}^{n}\left(s_{j}+\frac{d}{4}(n-1)\right) \alpha_{j}(-\mathbf{s}-\nu) \frac{\Gamma_{\Omega}(\mathbf{s}+\rho+\nu)}{\Gamma_{\Omega}\left(\mathbf{s}+\nu+\rho-\varepsilon_{j}\right)}\left(\mathcal{L}_{\nu} \varphi_{\mathbf{s}-\varepsilon_{j}}\right)(z) \\
& \sum_{j=1}^{n}\left(s_{j}+\nu-\frac{d}{4}(n-1)\right) \alpha_{j}(\mathbf{s}+\nu) \frac{\Gamma(\mathbf{s}+\rho+\nu)}{\Gamma_{\Omega}\left(\mathbf{s}+\nu+\rho+\varepsilon_{j}\right)}\left(\mathcal{L}_{\nu} \varphi_{\mathbf{s}+\varepsilon_{j}}\right)(z),
\end{aligned}
$$

and

$$
\begin{aligned}
& \frac{\Gamma_{\Omega}(\mathbf{s}+\rho+\nu)}{\Gamma_{\Omega}\left(\mathbf{s}+\nu+\rho-\varepsilon_{j}\right)}=\frac{\Gamma\left(s_{j}+\nu-\frac{d}{4}(n-1)\right)}{\Gamma\left(s_{j}+\nu-1-\frac{d}{4}(n-1)\right)}=s_{j}+\nu-1-\frac{d}{4}(n-1), \\
& \frac{\Gamma_{\Omega}(\mathbf{s}+\rho+\nu)}{\Gamma_{\Omega}\left(\mathbf{s}+\nu+\rho+\varepsilon_{j}\right)}=\frac{\Gamma\left(s_{j}+\nu-\frac{d}{4}(n-1)\right)}{\Gamma\left(s_{j}+\nu+1-\frac{d}{4}(n-1)\right)}=\frac{1}{s_{j}+\nu-\frac{d}{4}(n-1)} .
\end{aligned}
$$


Finally one observes that $\alpha_{j}(\mathbf{s}+\nu)=\alpha_{j}(\mathbf{s})$.

End of the proof of Theorem 5.2

(i) Let $D_{\nu}^{(4)}$ be the operator defined by the relation $D_{\nu}^{(4)} \mathcal{F}_{\nu}=\mathcal{F}_{\nu} D_{\nu}^{(3)}$. We will show that $D_{\nu}^{(4)}=D_{\nu}$. The modified spherical Fourier transform $\mathcal{F}_{\nu}$ is given by

$$
\left(\mathcal{F}_{\nu} \psi\right)(\mathbf{s})=\frac{1}{\Gamma_{\Omega}\left(\mathbf{s}+\frac{\nu}{2}+\rho\right)} \int_{\Omega} \varphi_{\mathbf{s}}(u) \psi(u) \Delta(u)^{\frac{\nu}{2}-\frac{N}{n}} m(d u) .
$$

Using the fact that the operator $D_{\nu}^{(3)}$ is symmetric with respect to the measure $\Delta(u)^{\nu-\frac{N}{n}} m(d u)$, one obtains

$$
\begin{aligned}
& D_{\nu}^{(4)}\left(\mathcal{F}_{\nu} \psi\right)(\mathbf{s})=\mathcal{F}_{\nu}\left(D_{\nu}^{(3)} \psi\right)(\mathbf{s}) \\
& =\frac{1}{\Gamma_{\Omega}\left(\mathbf{s}+\frac{\nu}{2}+\rho\right)} \int_{\Omega} \varphi_{\mathbf{s}-\frac{\nu}{2}}(u)\left(D_{\nu}^{(3)} \psi\right)(u) \Delta(u)^{\nu-\frac{N}{n}} m(d u) \\
& =\frac{1}{\Gamma_{\Omega}\left(\mathbf{s}+\frac{\nu}{2}+\rho\right)} \int_{\Omega}\left(D^{(3)} \varphi_{\mathbf{s}-\frac{\nu}{2}}\right)(u) \psi(u) \Delta(u)^{\nu-\frac{N}{n}} m(d u) .
\end{aligned}
$$

From Lemma 5.8 it follows that

$$
\begin{aligned}
& D_{\nu}^{(4)}\left(\mathcal{F}_{\nu} \psi\right)(\mathbf{s})= \\
& -\frac{1}{\Gamma_{\Omega}\left(\mathbf{s}+\frac{\nu}{2}+\rho\right)} \sum_{j=1}^{n}\left(s_{j}-\frac{\nu}{2}+\frac{d}{4}(n-1)\right)\left(s_{j}+\frac{\nu}{2}-1-\frac{d}{4}(n-1)\right) \\
& \alpha_{j}(-\mathbf{s}) \int_{\Omega} \varphi_{\mathbf{s}-\varepsilon_{j}}(u) \psi(u) \Delta^{\frac{\nu}{2}-\frac{N}{n}}(u) m(d u) \\
& +\frac{1}{\Gamma_{\Omega}\left(\mathbf{s}+\frac{\nu}{2}+\rho\right)} \sum_{j=1}^{n} \alpha_{j}(s) \int_{\Omega} \varphi_{\mathbf{s}+\varepsilon_{j}}(u) \psi(u) \Delta^{\frac{\nu}{2}-\frac{N}{n}}(u) m(d u) \\
& =-\sum_{j=1}^{n}\left(s_{j}-\frac{\nu}{2}+\frac{d}{4}(n-1)\right)\left(s_{j}+\frac{\nu}{2}-1-\frac{d}{4}(n-1)\right) \alpha_{j}(-\mathbf{s}) \\
& \frac{\Gamma_{\Omega}\left(\mathbf{s}+\frac{\nu}{2}-\varepsilon_{j}+\rho\right)}{\Gamma_{\Omega}\left(\mathbf{s}+\frac{\nu}{2}+\rho\right)}\left(\mathcal{F}_{\nu} \psi\right)\left(\mathbf{s}-\varepsilon_{j}\right) \\
& +\sum_{j=1}^{n} \alpha_{j}(\mathbf{s}) \frac{\Gamma_{\Omega}\left(\mathbf{s}+\frac{\nu}{2}+\varepsilon_{j}+\rho\right)}{\Gamma_{\Omega}\left(\mathbf{s}+\frac{\nu}{2}+\rho\right)}\left(\mathcal{F}_{\nu} \psi\right)\left(\mathbf{s}+\varepsilon_{j}\right) .
\end{aligned}
$$

As we saw in the proof of Lemma 5.8,

$$
\begin{aligned}
& \frac{\Gamma_{\Omega}\left(\mathbf{s}+\frac{\nu}{2}+\rho-\varepsilon_{j}\right)}{\Gamma_{\Omega}\left(\mathbf{s}+\rho+\frac{\nu}{2}\right)}=\frac{1}{s_{j}+\frac{1}{2} \nu-1-\frac{d}{4}(n-1)}, \\
& \frac{\Gamma_{\Omega}\left(\mathbf{s}+\frac{\nu}{2}+\rho+\varepsilon_{j}\right)}{\Gamma_{\Omega}\left(\mathbf{s}+\rho+\frac{\nu}{2}\right)}=s_{j}+\frac{1}{2} \nu-\frac{d}{4}(n-1) .
\end{aligned}
$$


This shows that $D_{\nu}^{(4)}=D_{\nu}$. Since the Laguerre functions $\Psi_{\mathbf{m}}^{(\nu)}$ are eigenfunctions of the operator $D_{\nu}^{(3)}$, hence the Meixner-Pollaczek polynomials $Q_{\mathbf{m}}^{(\nu)}$ are eigenfunctions of the operator $D_{\nu}^{(4)}=D_{\nu}$ :

$$
D_{\nu} Q_{\mathbf{m}}^{(\nu)}=(2|\mathbf{m}|+\nu) Q_{\mathbf{m}}^{(\nu)} .
$$

(ii) The identity in Theorem 3.1 can be written, with $\mathbf{s}=\mathbf{m}+\frac{\nu}{2}-\rho$,

$$
\sum_{\mathbf{k}} Q_{\mathbf{k}}^{(\nu)}\left(\mathbf{m}+\frac{\nu}{2}-\rho\right) \Phi_{\mathbf{k}}(w)=\Delta(e-w)^{-\nu} \Phi_{\mathbf{m}}\left((e-w)(e+w)^{-1}\right)=(-1)^{|\mathbf{m}|} 2^{-\nu} F_{\mathbf{m}}^{(\nu)}(w) .
$$

The right handside is an eigenfunction of $D_{\nu}^{(2)}$ for the eigenvalue $2|\mathbf{m}|+\nu$. The action of $D_{\nu}^{(2)}$ on $\Phi_{\mathbf{k}}$ has been computed (Lemma 5.7), hence

$$
\begin{aligned}
& (2|\mathbf{m}|+\nu) \sum_{\mathbf{k}} d_{\mathbf{m}} Q_{\mathbf{m}}^{(\nu)}\left(\mathbf{m}+\frac{\nu}{2}-\rho\right) \Phi_{\mathbf{m}}(w) \\
& =\sum_{\mathbf{k}} d_{\mathbf{k}} Q_{\mathbf{k}}^{(\nu)}\left(\mathbf{m}+\frac{\nu}{2}-\rho\right)\left(\sum_{j=1}^{r}\left(k_{j}+\nu-\frac{d}{2}(j-1)\right) \alpha_{j}(\mathbf{k}-\rho) \Phi_{\mathbf{k}+\varepsilon_{j}}(w)\right. \\
& \left.-\sum_{j=1}^{n}\left(k_{j}+\frac{d}{2}(n-j)\right) \alpha_{j}(-\mathbf{k}+\rho) \Phi_{\mathbf{k}-\varepsilon_{j}}(w)\right)
\end{aligned}
$$

By equaling the coefficients of $\Phi_{\mathbf{k}}(w)$ in both handsides, one gets statement (ii), observing that $2|\mathbf{m}|+\nu=2 \sum_{j=1}^{n} s_{j}$, since $\sum_{j=1}^{n} \rho_{j}=0$.

As in the one variable case, the operators $D_{\nu}^{(i)}(i=1,2,3,4)$ are essentially selfadjoint (with apporpriate domains).

6. A Barnes type integral. - Let $\mu$ be a positive measure on $\mathbb{R}$ with finite moments of all orders: for every $m \geq 0$,

$$
\int_{\mathbb{R}}|t|^{m} \mu(d t)<\infty
$$

For $d>0$ one defines the following probability measure on $\mathbb{R}^{n}$ :

$$
\frac{1}{Z_{n}^{(d)}}\left|V\left(x_{1}, \ldots, x_{n}\right)\right|^{d} \mu\left(d x_{1}\right) \ldots \mu\left(d x_{n}\right),
$$

with

$$
Z_{n}^{(d)}=\int_{\mathbb{R}^{n}}\left|V\left(x_{1}, \ldots, x_{n}\right)\right|^{d} \mu\left(d x_{1}\right) \ldots \mu\left(d x_{n}\right) .
$$

A general problem is to evaluate its moments:

$$
\mathfrak{M}(\chi)=\frac{1}{Z_{n}^{(d)}} \int_{\mathbb{R}^{n}} \chi\left(x_{1}, \ldots, x_{n}\right)\left|V\left(x_{1}, \ldots, x_{n}\right)\right|^{d} \mu\left(d x_{1}\right) \ldots \mu\left(d x_{n}\right),
$$


where $\chi$ is a symmetric polynomial. Special attention have been made to the case

$$
F_{1}(z)=\frac{1}{Z_{n}^{(d)}} \int_{\mathbb{R}^{n}} \prod_{j=1}^{n}\left(z-x_{j}\right)\left|V\left(x_{1}, \ldots, x_{n}\right)\right|^{d} \mu\left(d x_{1}\right) \ldots \mu\left(d x_{n}\right),
$$

and, more generally,

$$
F_{K}\left(z_{1}, \ldots, z_{K}\right)=\frac{1}{Z_{n}^{(d)}} \int_{\mathbb{R}^{n}} \prod_{k=1}^{K} \prod_{j=1}^{n}\left(z_{k}-x_{j}\right)\left|V\left(x_{1}, \ldots, x_{n}\right)\right|^{d} \mu\left(d x_{1}\right) \ldots \mu\left(d x_{n}\right) .
$$

For $d=2$ the use of orthogonal polynomials leads to the evaluation of $F_{1}(z)$ and $F_{K}\left(z_{1}, \ldots, z_{K}\right)$. Let $\left(p_{m}\right)$ be the sequence of orthogonal polynomials with respect to the measure $\mu$, normalized by the condition

$$
p_{m}(t)=t^{m}+\text { lower order terms. }
$$

Then

$$
Z_{n}^{(d)}=n ! h_{0} h_{1} \ldots h_{n-1},
$$

where

$$
h_{m}=\int_{\mathbb{R}} p_{m}(t)^{2} \mu(d t) .
$$

Proposition 6.1. - (i) For $z \in \mathbb{C}$,

$$
F_{1}(z)=p_{n}(z) .
$$

(ii) For $z_{1}, \ldots, z_{K} \in \mathbb{C}$,

$$
F_{K}\left(z_{1}, \ldots, z_{K}\right)=\frac{\operatorname{det}\left(p_{n+j-1}\left(z_{k}\right)\right)_{1 \leq j, k \leq K}}{V\left(z_{1}, \ldots, z_{K}\right)} .
$$

(See [Szegö,1975] p.27, formula (2.2.10) for (i), and [Brézin-Hikami,2000] for (ii). In point of fact (i) appeared already in [Heine,1878].)

If $\mu$ is the measure on $\mathbb{R}$ given by

$$
\int_{\mathbb{R}} f(t) \mu(d t)=\int_{-1}^{1} f(t)(1-t)^{\alpha}(1+t)^{\beta} d t
$$

with $\alpha, \beta>-1$, then $Z_{n}^{(d)}$ is the Selberg integral and the evaluation of

$$
\mathfrak{M}(\chi)=\frac{1}{Z_{n}^{(d)}} \int_{\mathbb{R}^{n}} \chi\left(x_{1}, \ldots, x_{n}\right)\left|V\left(x_{1}, \ldots, x_{n}\right)\right|^{d} \prod_{j=1}^{n}\left(1-x_{i}\right)^{\alpha}\left(1+x_{i}\right)^{\beta} d x_{1} \ldots d x_{n}
$$

has been considered by Aomoto, Kaneko, and Mimachi. 
Proposition 6.2. - (i) For $z \in \mathbb{C}$,

$$
F_{1}(z)=C p_{n}^{\left(\frac{2}{d} \alpha, \frac{2}{d} \beta\right)}(z)
$$

where $p_{m}^{(\alpha, \beta)}$ is the Jacobi polynomial of degree $m$.

(ii) For $z_{1}, \ldots, z_{K} \in \mathbb{C}$,

$$
F_{K}\left(z_{1}, \ldots, z_{K}\right)=C P_{\left(n^{K}\right)}^{\left(\frac{2}{d} \alpha, \frac{2}{d} \beta, \frac{2}{d}\right)}\left(z_{1}, \ldots, z_{K}\right),
$$

where $P_{\mathbf{m}}^{(\alpha, \beta, \gamma)}$ is the multivariate Heckman-Opdam Jacobi polynomial for the partition $\mathbf{m}=n^{K}=(n, \ldots, n)$.

([Aomoto,1987] for (i), [Kaneko,1993], [Mimachi,2001] for (ii).)

For $d=2$, formulae (i) and (ii) agree with formulae (i) and (ii) in Proposition 6.1. In fact, for $d=2$, there is a determinantal formula for the multivariate Heckman-Opdam Jacobi polynomials.

Mimachi considered the following Barnes type integral, for $\kappa>0$,

$$
\mathfrak{M}(\chi)=\frac{1}{Z_{n}^{(d)}} \int_{\mathbb{R}^{n}} \chi\left(\lambda_{1}, \ldots, \lambda_{n}\right) \prod_{j=1}^{n}\left|\Gamma\left(i \lambda_{j}+\frac{\kappa}{2}\right)\right|^{2} \frac{1}{|c(i \lambda)|^{2}} d \lambda_{1} \ldots d \lambda_{n}
$$

where $\chi$ is a symmetric polynomial, and

$$
Z_{n}^{(d)}=\int_{\mathbb{R}^{n}} \prod_{j=1}^{n}\left|\Gamma\left(i \lambda_{j}+\frac{\kappa}{2}\right)\right|^{2} \frac{1}{|c(i \lambda)|^{2}} d \lambda_{1} \ldots d \lambda_{n} .
$$

Proposition 6.3. - Define

$$
F_{1}(z)=\frac{1}{Z_{n}^{(d)}} \int_{\mathbb{R}^{n}} \prod_{j=1}^{n}\left(z-\lambda_{j}\right) \prod_{j=1}^{n}\left|\Gamma\left(i \lambda_{j}+\frac{\kappa}{2}\right)\right|^{2} \frac{1}{|c(i \lambda)|^{2}} d \lambda_{1} \ldots d \lambda_{n}
$$

Then

$$
F_{1}(z)=i^{n} n !\left(\frac{d}{4}\right)^{n} q_{n}^{\left(\frac{2}{d} \kappa\right)}\left(\frac{2}{d} i z\right),
$$

where $q_{m}^{(\nu)}$ is the one variable Meixner-Pollaczek polynomial of degree $m$.

([Mimachi,1999]. Actually this is a special case of Mimachi's result: $\phi=\frac{\pi}{2}, \lambda=\frac{d}{2}$ with Mimachi's notation.)

In case $d=2$, the reciprocal of the $c$-function is the Vandermonde polynomial:

$$
\frac{1}{|c(i \lambda)|^{2}}=c_{0}^{2} V\left(\lambda_{1}, \ldots, \lambda_{n}\right)^{2} .
$$


Then Proposition 6.3 is a special case of (i) in Proposition 6.1.

We propose an alternative proof of Proposition 6.3. In our setting Mimachi's integral can be written

$$
\mathfrak{M}(\chi)=a_{\nu}^{(4)} \int_{\mathbb{R}^{n}} \chi(\lambda)\left|\Gamma_{\Omega}\left(i \lambda+\rho+\frac{\nu}{2}\right)\right|^{2} \frac{1}{|c(i \lambda)|^{2}} d \lambda_{1} \ldots d \lambda_{n},
$$

with $\nu=\kappa+\frac{d}{2}(n-1)$. Recall that the map

$$
\gamma: \mathbb{D}(\Omega) \rightarrow \mathcal{P}\left(\mathbb{C}^{n}\right)^{\mathfrak{S}_{n}}, \quad D \mapsto \gamma_{D}
$$

is an isomorphism. Therefore, given $\chi \in \mathcal{P}\left(\mathbb{C}^{n}\right)^{\mathfrak{S}_{n}}$, there is an invariant differential operator $D$ such that

$$
\chi(\lambda)=\gamma_{D}\left(-i \lambda-\frac{\nu}{2}\right)
$$

and, by Lemma 2.1, $\chi$ is also the modified spherical Fourier transform of $D \Psi_{0}\left(\Psi_{0}(u)=\right.$ $\left.e^{-\operatorname{tr}(u)}\right)$. Therefore, by the Plancherel formula,

$$
\mathfrak{M}(\chi)=a_{\nu}^{(3)} \int_{\Omega}\left(D \Psi_{0}\right)(u) \Psi_{0}(u) \Delta(u)^{\nu-\frac{N}{n}} m(d u)
$$

a) If $\chi(\lambda)=\gamma_{\mathbf{m}}\left(-i \lambda-\frac{\nu}{2}\right)$, then $D=D^{\mathbf{m}}$, the invariant differential operator such that $\sigma_{D}(u, e)=\Phi_{\mathbf{m}}(u)$, and $D \Psi_{0}(u)=\Psi_{\mathbf{m}}(-u) e^{-\operatorname{tr}(u)}$. In that case

$$
\mathfrak{M}(\chi)=(-1)^{|\mathbf{m}|} a_{\nu}^{(3)} \int_{\Omega} \Phi_{\mathbf{m}}(u) e^{-2 \operatorname{tr}(u)} \Delta(u)^{\nu-\frac{N}{n}} d u=(-1)^{|\mathbf{m}|} 2^{-|\mathbf{m}|}(\nu)_{\mathbf{m}}
$$

b) We consider now the invariant differential operator $D_{\alpha}(\alpha \in \mathbb{C})$ :

$$
D_{\alpha}=\Delta(u)^{1+\alpha} \Delta\left(\frac{\partial}{\partial u}\right) \Delta(u)^{-\alpha}
$$

(See [Faraut-Korányi,1994] p. 294.) The polynomial $\gamma_{D^{\alpha}}$ has been computed (p.296):

$$
\gamma_{D^{\alpha}}(\mathbf{s})=\prod_{j=1}^{n}\left(s_{j}-\alpha+\frac{d}{4}(n-1)\right)
$$

and by Proposition XIV.1.5,

$$
D_{\alpha}=\sum_{k=0}^{n}(-1)^{k}\left(\begin{array}{l}
n \\
k
\end{array}\right)(\alpha)_{\langle k\rangle} D^{\langle n-k\rangle}
$$


$(\langle k\rangle$ denotes the partition $(1, \ldots, 1,0, \ldots, 0)$ with 1 repeated $k$ times $)$. Therefore, by a),

$$
\mathfrak{M}(\chi)=(-1)^{n} 2^{-n} \sum_{k=1}^{n}\left(\begin{array}{l}
n \\
k
\end{array}\right)(\alpha)_{\langle k\rangle}(\nu)_{\langle n-k\rangle} 2^{k}
$$

The coefficient $(\alpha)_{\langle k\rangle}$ can be written:

$$
(\alpha)_{\langle k\rangle}=\alpha\left(\alpha-\frac{d}{2}\right)\left(\alpha-2 \frac{d}{2}\right) \ldots\left(\alpha-(k-1) \frac{d}{2}\right)=\left(\frac{d}{2}\right)^{k}\left[\frac{2}{d} \alpha\right]_{k}
$$

Hence

$$
\mathfrak{M}(\chi)=(-1)^{n}\left(\frac{d}{4}\right)^{n} \sum_{k=0}^{n}\left(\begin{array}{l}
n \\
k
\end{array}\right)\left[\frac{2}{d} \alpha\right]_{k}\left[\frac{2}{d} \nu\right]_{n-k} 2^{k}
$$

By observing that

$$
\begin{aligned}
\sum_{k=0}^{n}\left(\begin{array}{l}
n \\
k
\end{array}\right)[u]_{k}[v]_{n-k} 2^{k} & =[v]_{n} \sum_{k=0}^{n}\left(\begin{array}{l}
n \\
k
\end{array}\right)(-1)^{k}(-u)_{k} \frac{1}{(v-n+1)_{k}} 2^{k} \\
& =n ! q_{n}^{(v-n+1)}\left(-u-\frac{v-n+1}{2}\right)
\end{aligned}
$$

as we saw in the proof of Proposition 1.1, we obtain the result.

A natural question arises: is it possible to evaluate the integral

$$
\left.F_{K}\left(z_{1}, \ldots, z_{K}\right)\right)=\frac{1}{Z_{n}^{(d)}} \int_{\mathbb{R}^{n}} \prod_{k=1}^{K} \prod_{j=1}^{n}\left(z_{k}-\lambda_{j}\right) \prod_{j=1}^{n}\left|\Gamma\left(i \lambda_{j}+\frac{\kappa}{2}\right)\right|^{2} \frac{1}{|c(i \lambda)|^{2}} d \lambda_{1} \ldots d \lambda_{n}
$$

in terms of the multivariate Meixner-Pollaczek polynomials $Q_{\left(n^{K}\right)}^{(\nu)}\left(\zeta_{1}, \ldots, \zeta_{k}\right)$ ? For $d=2$, by (ii) in Proposition 6.1 and Theorem 4.3, it holds:

$$
F_{K}\left(z_{1}, \ldots, z_{K}\right)=C Q_{\left(n^{K}\right)}^{(\kappa)}\left(i z_{1}, \ldots, i z_{k}\right)
$$

Andrews, G. E., Askey, R. And Roy, R. (1999). Special functions. Cambridge.

Aомото, K. (1987). Jacobi polynomials associated with Selberg integrals, SIAM J. Math. Anal., 18, 545-549.

Aristidou, M., Davidson, M., Ólafsson, G. (2006). Differential recursion relations for Laguerre functions on symmetric cones, Bull. Sci. Math., 130, 246-263.

Aristidou, M., Davidson, M., Ólafsson, G. (2007). Laguerre functions on symmetric cones and recursion relations in the real case, J. Comput. Appl. Math., 199, 95-112.

Brézin, É., Hikami, S. (2000). Characteristic polynomials of random matrices, Commun. Math. Physics, 214, 111-135. 
Bump, D, Choi, K-K., Kurlberg, P. and Vaaler, J. (2000). A local Riemann hypothesis, I, Math. Z., 233, 1-19.

ChÉbli, H., Faraut, J. (2004). Fonctions holomorphes à croissance modérée et vecteurs distributions, Math. Z., 248, 541-565.

Davidson, M., Ólafsson, G., Zhang, G. (2003). Laplace and Segal-Bargmann transforms on Hermitian symmetric spaces and orthogonal polynomials, J. Functional Analysis, 204, 157-195.

Davidson, M., Ólafsson, G. (2003). Differential recursion relations for Laguerre functions on Hermitian matrices, Integral transforms and special functions, 14, 469-484.

DiB, H. (1990). Fonctions de Bessel sur une algèbre de Jordan, J. Math. pures et appl., 69, 403-448.

Faraut, J. (2004). Loi du demi-cercle de Wigner et polynômes de Laguerre. Preprint, to appear in Sém. \& Congrès, Soc. Math. France.

Faraut, J., Korányi, A. (1994). Analysis on symmetric cones. Oxford.

HaAgerup, U., Thorbuørnsen, S. (2003). Random matrices with complex Gaussian entries, Exp. Mat., 21, 293-337.

HARer, J., ZAGier, D. (1986). The Euler characteristic of the moduli space of curves, Invent. Math., 85, 457-485.

Heine, E. (1878). Handbuch der Kugelfunktionen. Vol.I.

HUA, L.K. (1963). Harmonic analysis of functions of several variables in the classical domains. Amer. Math. Soc..

KAneKo, J. (1993). Selberg integrals and hypergeometric functions associated with Jack polynomials, SIAM J. Math. Anal., 24, 1086-1110.

Kutznetsov, A. (2008). Expansion of the Riemann $\Xi$-function in Meixner-Pollaczek polynomials, Canad. Math. Bull., XX, 1-9.

Lassalle, M. (1998). Coefficients binomiaux généralisés et polynômes de Macdonald, $J$. Funct. Analysis, 158, 289-324.

Menta, M. L. (1991). Random matrices. Academic Press.

Mimachi, K. (2001). A duality of Macdonald-Koornwinder polynomials and its application to integral representations, Duke Math. J., 107, 265-281.

Mimachi, K. (1999a). Barnes-type integral and the Meixner-Pollaczek polynomials, Letters in Math. Physics, 48, 365-373.

Mimachi, K. (1999b). A multidimensional generalization of the Barnes integral and the continuous Hahn polynomial, J. Math. Anal. Appl., 234, 67-76.

Okunkov, A., Olshanski, G. (1997). Shifted Jack polynomials, binomial formula, and applications, Math. Res. Letters, 4, 69-78.

Ørsted, B., Zhang, G. (1994). Weyl quantization and tensor products of Fock and Bergman spaces, Indiana Univ. Math. J., 43, 551-583.

Ørsted, B., Zhang, G. (1995). Generalized principal series representations and tube domains, Duke Math. J., 78, 335-357.

Ricci, F., Tabacco Vignati A. (1994). Bergman spaces on some tube-type domains and Laguerre operators on symmetric cones, J. Reine Angew. Math., 449, 81-101.

SAHI, S., ZhANG, G. (2007). Biorthogonal expansion of non-symmetric Jack functions, SIGMA, 3, Paper 106. 
SzeGö, G. (1975). Orthogonal polynomials. Amer. Math. Soc.

ZHANG, G. (1995). Some recurrence formulas for spherical polynomials on tube domains, Trans. Amer. Math. Soc., 347, 1725-1734.

\author{
Jacques Faraut \\ Institut de Mathématiques de Jussieu \\ Université Pierre et Marie Curie \\ 175 rue du Chevaleret, 75013 Paris, France \\ faraut@math.jussieu.fr \\ Masato Wakayama \\ Faculty of Mathematics \\ Kyushu University \\ Hakozaki, Fukuoka, 812-8581 Japan \\ wakayama@math.kyushu-u.ac.jp
}




\section{List of MI Preprint Series, Kyushu University}

The Grobal COE Program

Math-for-Industry Education \& Research Hub

MI

2008-1 Takahiro ITO, Shuichi INOKUCHI \& Yoshihiro MIZOGUCHI

Abstract collision systems simulated by cellular automata

2008-2 Eiji ONODERA

The intial value problem for a third-order dispersive flow into compact almost Hermitian manifolds

2008-3 Hiroaki KIDO

On isosceles sets in the 4-dimensional Euclidean space

2008-4 Hirofumi NOTSU

Numerical computations of cavity flow problems by a pressure stabilized characteristiccurve finite element scheme

2008-5 Yoshiyasu OZEKI

Torsion points of abelian varieties with values in nfinite extensions over a padic field

2008-6 Yoshiyuki TOMIYAMA

Lifting Galois representations over arbitrary number fields

2008-7 Takehiro HIROTSU \& Setsuo TANIGUCHI

The random walk model revisited

2008-8 Silvia GANDY, Masaaki KANNO, Hirokazu ANAI \& Kazuhiro YOKOYAMA Optimizing a particular real root of a polynomial by a special cylindrical algebraic decomposition

2008-9 Kazufumi KIMOTO, Sho MATSUMOTO \& Masato WAKAYAMA

Alpha-determinant cyclic modules and Jacobi polynomials 
2008-10 Sangyeol LEE \& Hiroki MASUDA

Jarque-Bera Normality Test for the Driving Lévy Process of a Discretely Observed Univariate SDE

2008-11 Hiroyuki CHIHARA \& Eiji ONODERA

A third order dispersive flow for closed curves into almost Hermitian manifolds

2008-12 Takehiko KINOSHITA, Kouji HASHIMOTO and Mitsuhiro T. NAKAO

On the $L^{2}$ a priori error estimates to the finite element solution of elliptic problems with singular adjoint operator

2008-13 Jacques FARAUT and Masato WAKAYAMA

Hermitian symmetric spaces of tube type and multivariate Meixner-Pollaczek polynomials 